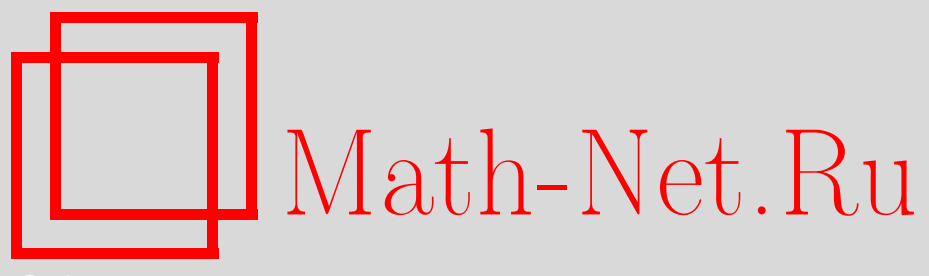

Е. Р. Аваков, Г. Г. Магарил-Ильяев, В. М. Тихомиров,

О принципе Лагранжа в задачах на экстремум при наличии ограничений, УМН, 2013, том 68, выпуск 3, 5-38

DOI: https://doi.org/10.4213/rm9525

Использование Общероссийского математического портала Math-Net.Ru подразумевает, что вы прочитали и согласны с пользовательским соглашением http://www . mathnet.ru/rus/agreement

Параметры загрузки:

IP : 54.162 .85 .209

26 апреля 2023 г., 15:58:05

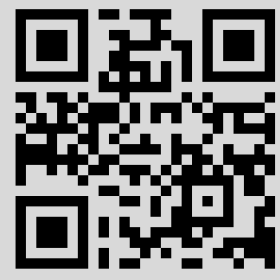




\section{О принципе Лагранжа в задачах на экстремум при наличии ограничений}

\section{Е. Р. Аваков, Г. Г. Магарил-Ильяев, В. М. Тихомиров}

В работе доказывается один общий результат о принципе Лагранжа для так называемых гладко-аппроксимативно-выпуклых задач, охватывающий необходимые условия экстремума для задач математического и выпуклого программирования, вариационного исчисления, ляпуновских задач и задач оптимального управления с фазовыми ограничениями. Рассмотрена также задача локальной управляемости динамической системы с фазовыми ограничениями. В дополнении приведены результаты, связанные с развитием "лагранжева подхода" к задачам, где отсутствует регулярность и где классические подходы оказываются бессодержательными.

Библиография: 33 названия.

Ключевые слова: экстремальная задача, оптимальное управление, фазовые ограничения, микс, управляемость, анормальность.

DOI: $10.4213 / \mathrm{rm} 9525$

\section{СоДЕРЖАНИЕ}

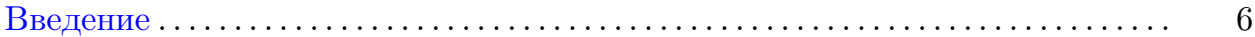

1. Формулировка основного результата ........................ 9

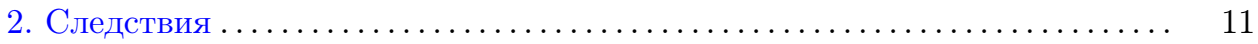

2.1. Математическое программирование................... 12

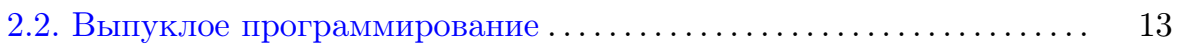

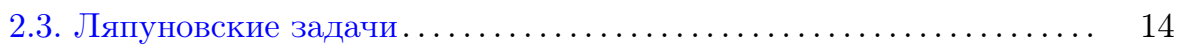

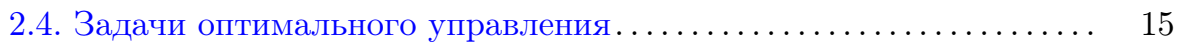

2.5. Задача локальной управляемости ...................... 21

3. Доказательство основной теоремы ....................... 23

4. Дополнение: дальнейшее развитие формализма Лагранжа (Е. Р. Аваков) 30

4.1. Введение . . . .................................. 30

4.2. Теорема о поправке .............................. 32

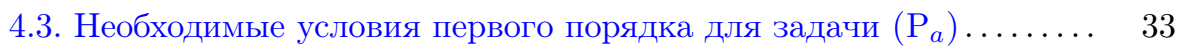

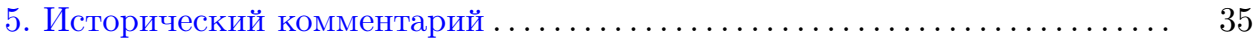

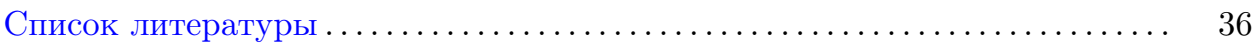

Работа выполнена при поддержке РФФИ (гранты № 10-01-00188, 11-01-00529).

(C) Е. Р. Аваков, Г. Г. МАГАРИЛ-ИЛЬЯЕв, В. М. ТихомиРов, 2013 


\section{Введение}

Интерес к экстремальным задачам, т. е. задачам на максимум и минимум, проявился уже на заре развития математики. Стимулом для древних исследователей были причины эстетического характера, стремление к совершенству, любознательность. Эти свойства присущи человеку во все времена, и поныне они дают поводы для поиска наилучших решений. Важной причиной, побуждающей исследовать экстремальные задачи, является также и то, что многие законы природы основываются на вариационных (экстремальных) принципах (по этому поводу Эйлер заметил: “В мире не происходит ничего, в чем не был бы виден смысл какого-нибудь максимума или минимума”). Нельзя не назвать также и прагматические причины, по которым необходимо решать экстремальные задачи. Человеку свойственно наилучшим образом использовать ресурсы, находящиеся в его распоряжении, и потому экстремальные задачи естественным образом возникают при управлении различными процессами, в экономике, инженерии.

Среди наиболее ранних точно решенных задач - так называемая изопериметрическая задача - задача о форме кривой заданной длины, охватывающей наибольшую площадь ${ }^{1}$, и задача о форме поверхности заданной площади, охватывающей наибольший объем. Ответы на эти задачи для мыслителей Древней Греции были символами совершенства человеческого разума. Крупнейшие их представители: Евклид, Архимед и Аполлоний ставили и решали различные геометрические задачи на экстремум. Задача о параллелограмме наибольшей площади, который можно вписать в треугольник, приводится в "Началах" Евклида (III в. до н. э.); задача о шаровом сегменте максимального объема при заданной площади шаровой части поверхности этого сегмента содержится в сочинениях Архимеда (тоже III в. до н.э.); задача о минимальном расстоянии от точки плоскости до эллипса и о нормалях $к$ эллипсу из прочзвольной точки плоскости была поставлена и решена Аполлонием (III-II в. до н. э.) в его знаменитых "Кониках". Множество задач, в основном также геометрического содержания, было решено в Новое время Вивиани, Торичелли, Кеплером и другими.

Первый экстремальный принцип в естествознании выдвинул Пьер Ферма (1662 г.). Согласно этому принципу, свет "избирает" такую траекторию, вдоль которой время, затрачиваемое им на преодоление пути от одной точки до другой, минималъно. Законы преломления света, установленные экспериментально, находятся, исходя из этого принципа, как решения соответствующих экстремальных задач.

Первой задачей инженерного содержания была так называемая аэродинамическая задача Нъютона о теле вращения заданной ширины и высоты, испытывающем наименьшее сопротивление в некоей "редкой среде", поставленная и решенная Ньютоном в 1687 г. в "Математических началах натуральной философии" [1].

До определенного времени каждая экстремальная задача решалась индивидуально, так сказать, без теории. Начала теории экстремума часто исчисляют

\footnotetext{
${ }^{1}$ Ответ в ней приводил в своих сочинениях еще Аристотель (IV в. до н. э.).
} 
от 1638 г., когда Ферма [2] написал письмо Декарту, в котором содержался намек на необходимое условие экстремума для гладкой функции одного переменного, заключающееся в том, что в точке экстремума главная линейная часть приращения функции равна нулю. Ньютон этот факт охарактеризовал весьма выразительно (на языке динамики, для него независимая переменная время): “когда величина является максимальной или минимальной, в этот момент она не течет ни вперед, ни назад”. Современное толкование наблюдению Ферма придали Ньютон и Лейбниц; оно состоит в том, что в точке локального экстремума гладкой функции производная этой функции равна нулю (теорема Ферма).

Затем, едва родившись, теория экстремума совершила неожиданный скачок “от единицы к бесконечности": возникли задачи, в которых требовалось найти минимум или максимум не функции одного переменного, а функционала, где сами функции являются переменными величинами. Это произошло в 1696 г., когда Иоганн Бернулли (1667-1748) в первом в истории научном журнале "Acta Eruditorum" опубликовал статью [3], озаглавленную "Новая задача, к разрешению которой приглашаются математики". В статье была поставлена задача о брахистохроне, т. е. задача о нахождении формы кривой, соединяющей две точки в вертикальной плоскости, вдоль которой тело под действием силы тяжести без трения проходит путь от одной точки до другой за кратчайшее время (постановка, возможно, была навеяна более ранними размышлениями Галилея на эту тему). На призыв И. Бернулли откликнулись его брат Я. Бернулли, его учитель Лейбниц, Лопиталь и Ньютон, при этом каждый из них решил задачу по-своему. Примерно через четверть века И. Бернулли поставил перед своим учеником Леонардом Эйлером (1707-1783) проблему нахождения общих методов решения задач, сходных с задачей о брахистохроне. Эйлер справился с этой проблемой и нашел аналог теоремы Ферма - необходимое условие экстремума для экстремальной кривой. Им оказалось дифференциальное уравнение, получившее название уравнения Эйлера. Итоги своих многолетних исследований в данном направлении Эйлер подвел в монографии [4], изданной в 1744 г.

В пятидесятые годы восемнадцатого столетия взошла звезда Жозефа-Луи Лагранжа (1736-1813), который, во-первых, разработал новый метод исследования задач, изучавшихся Эйлером, получивший название метода вариаций (что дало повод Эйлеру все направление назвать вариационным исчислением), а во-вторых, описал прием исследования задач с ограничениями, который стали называть правилом множителей Лагранжа [5]. Лагранж внес огромный вклад и в осознание того, что вариационное исчисление является основой многих глав естествознания. Само вариационное исчисление интенсивно развивалось вплоть до сороковых годов двадцатого столетия (некоторые итоги были подведены в монографии [6]).

В конце тридцатых годов родилось новое направление в теории экстремума, которое было стимулировано потребностями математического осмысления процессов и явлений экономики. Оно имеет дату своего рождения - 1939 год, когда вышла брошюра Леонида Витальевича Канторовича (1912-1986) [7]. Это направление получило название линейного программирования и связано с изучением задач минимизации (или максимизации) линейных функций на выпук- 
лых многогранниках. Затем, в сороковые годы, данное направление было поглощено разделом теории экстремума, которое стали называть выпуклым программированием. Здесь изучаются задачи минимизации выпуклых функций на выпуклых множествах, где сами множества задаются, как правило, функциональными ограничениями типа неравенств. В те же времена стали изучаться и гладкие задачи с ограничениями типа равенств и неравенств (необходимые условия экстремума для всех этих классов задач были получены Карушем, Джоном, Куном и Таккером и др. [8]).

Замечательная теорема Алексея Андреевича Ляпунова (1912-1973) [9] о векторных мерах позволила многие задачи с интегральными функционалами (так называемые ляпуновские задачи) рассматривать как задачи выпуклого программирования.

В сороковые годы возникло еще одно направление в теории экстремальных задач, вызванное разнообразными проблемами управления (производственными процессами, космической навигацией и т. п.). Оно получило название onтимального управления. Начала теории необходимых условий для проблем оптимального управления были построены Львом Семеновичем Понтрягиным (1908-1988) и его коллегами [10] и развиты во многих направлениях (см., например, [11]).

Выше было сказано, что для гладких задач (с ограничениями типа равенств) Лагранж предложил метод их решения. Вот что он сам сказал по этому поводу: "Можно высказать следующий общий принцип. Если ищется максимум или минимум некоторой функиии многих переменных при условии, что между этими переменными имеется связь, задаваемая одним или несколькими уравнениями, нужно прибавить к функции, о которой говорилось, функици, задающие уравнения связи, умноженные на неопределенные множители, и искать затем максимум и минимум построенной суммы, как если бы переменные были независимы. Полученные уравнения, присоединенные к уравнениям связи, послужат для определения всех неизвестных". Иначе говоря, необходимое условие экстремума для таких задач состоит в том, что в точке локального экстремума в гладкой задаче с ограничениями типа равенств должно удовлетворяться необходимое условие экстремума построенной суммъ (которая теперь называется функцией Лагранжа) в задаче без ограничений.

При работе над книгой "Теория экстремальных задач" [12] ее авторы обнаружили, что необходимые условия экстремума в задачах математического и выпуклого программирования, вариационного исчисления, ляпуновских задачах, задачах оптимального управления и во многих других экстремальных задачах (в частности, условия, полученные Ферма, Эйлером, Лагранжем, Лежандром, Якоби, и их обобщения вплоть до Блисса, условия Каруша-Джона-Куна-Таккера, принцип максимума Понтрягина, условия, полученные Р. В. Гамкрелидзе, А.Я. Дубовицким и А.А. Милютиным и многими другими исследователями) соответствуют общему правилу, которое было названо приниипом Лагранжа: необходимые условия в задаче с ограничениями суть необходимые условия минимума функции Лагранжа “как если бы переменные были независимы”.

Естественно желание выяснить причину такой универсальности принципа Лагранжа. В книге [12] было показано, что весьма большой пласт необходи- 
мых условий экстремума покрывается одним результатом, касающимся задач, в которых переплетены гладкая и выпуклая структуры. Такие задачи были названы гладко-выпуклыми. Для них доказывалась одна теорема о принципе Лагранжа (иногда включавшаяся и в стандартный обязательный курс оптимизации на мехмате МГУ), которая позволяла в качестве следствия выводить необходимые условия экстремума в задачах математического и выпуклого программирования, а с ними - в задачах вариационного исчисления и линейных по фазе задачах оптимального управления. Но она не охватывала в полной мере задачи оптимального управления, и для вывода из нее принципа максимума Понтрягина требовались специальные ухищрения. Суть дела в том, что в таких задачах, вообще говоря, нет выпуклости, а имеется лишь так называемая "аппроксимативная выпуклость".

Цель работы - доказать одну общую теорему, утверждающую, что принцип Лагранжа верен для достаточно широкого класса экстремальных задач, называемых здесь гладко-аппроксимативно-выпуклыми задачами. Эта теорема объединяет результаты о необходимых условиях экстремума для перечисленных классов задач и вскрывает истинные причины "лагранжева единства" в теории необходимых условий, в основе которого лежат гладкость и (обычно скрытая) выпуклость, порождаемая интегральными операторами и ограничениями типа неравенств.

Статья состоит из трех основных разделов, дополнения и краткого исторического комментария. В первом разделе формулируется основной результат принцип Лагранжа для гладко-аппроксимативно-выпуклых задач. Во второмвыводятся различные следствия из этой теоремы: необходимые условия минимума в задачах математического и выпуклого программирования, ляпуновских задачах, задачах оптимального управления с фазовыми ограничениями, а также рассматривается задача локальной управляемости динамической системы с фазовыми ограничениями. В третьем разделе доказывается основная теорема. В дополнении представлены результаты первого из авторов, посвященные развитию “лагранжева подхода" к так называемым анормальным задачам, в которых отсутствует регулярность и поэтому классические подходы оказываются бессодержательными.

\section{1. Формулировка основного результата}

Пусть $X, Y_{1}$ и $Y_{2}$ - нормированные пространства, $\mathscr{U}$ - топологическое пространство, $V$ - открытое подмножество $X, f_{0}: V \times \mathscr{U} \rightarrow \mathbb{R}, F: V \times \mathscr{U} \rightarrow Y_{1}$, $G: V \rightarrow Y_{2}$ и $Q$ - непустое подмножество $Y_{2}$. Рассмотрим задачу

$$
f_{0}(x, u) \rightarrow \min , \quad F(x, u)=0, \quad G(x) \in Q .
$$

Пара $(x, u) \in V \times \mathscr{U}$ называется допустимой в задаче $(\mathrm{P})$, если $F(x, u)=0$ и $G(x) \in Q$. Допустимая пара $(\widehat{x}, \widehat{u})$ называется сильным (локальным) минимумом в задаче $(\mathrm{P})$, если найдется такое $\varepsilon>0$, что для любой допустимой пары $(x, u)$, для которой $\|x-\widehat{x}\|_{X}<\varepsilon$, выполняется неравенство $f_{0}(x, u) \geqslant f_{0}(\widehat{x}, \widehat{u})$.

Гладкая аппроксимативная выпуклость задачи $(\mathrm{P})$ означает, попросту говоря, что соответствующие отображения "гладкие" по переменной $x$ и "почти выпуклые" по переменной $u$. Приведем точные определения. 
Для натурального $m$ обозначим $\mathbb{R}_{+}^{m}=\left\{\alpha=\left(\alpha_{1}, \ldots, \alpha_{m}\right) \in \mathbb{R}^{m} \mid \alpha_{i} \geqslant 0, i=\right.$ $1, \ldots, m\}, \Sigma^{m}=\left\{\alpha=\left(\alpha_{1}, \ldots, \alpha_{m}\right) \in \mathbb{R}_{+}^{m} \mid \sum_{i=1}^{m} \alpha_{i}=1\right\}$, и пусть $e_{1}=(1,0, \ldots, 0)$, $\ldots, e_{m}=(0, \ldots, 0,1)-$ стандартный базис в $\mathbb{R}^{m}$.

Пусть $X, Z$ - нормированные пространства, $\mathscr{U}$ - топологическое пространство, $V$ - окрестность точки $\widehat{x} \in X$ и $\widehat{u} \in \mathscr{U}$. Скажем, что отображение $\Phi: V \times \mathscr{U} \rightarrow Z$ гладко-аппроксимативно-выпукло в точке $(\widehat{x}, \widehat{u})$, если оно

(а) аппроксимативно-выпукло в $\widehat{x}$, т. е. для любого набора $\bar{u}=\left(u_{1}, \ldots, u_{m}\right) \in$ $\mathscr{U}^{m}$, любых $\alpha=\left(\alpha_{1}, \ldots, \alpha_{m}\right) \in \Sigma^{m}$ и $\delta>0$ найдется такой элемент $M(\alpha, \delta, \bar{u}) \in$ $\mathscr{U}$ (называемый миксом $\left.u_{1}, \ldots, u_{m}\right)$, что $M\left(e_{i}, \delta, \bar{u}\right)=u_{i}, i=1, \ldots, m$, отображение $\alpha \mapsto M(\alpha, \delta, \bar{u})$ непрерывно на $\Sigma^{m}$ равномерно по $\delta$ и $\Phi(\widehat{x}, M(\alpha, \delta, \bar{u})) \rightarrow$ $\sum_{i=1}^{m} \alpha_{i} \Phi\left(\widehat{x}, u_{i}\right)$ при $\delta \rightarrow 0$ равномерно на $\Sigma^{m} ;^{2}$

(b) гладкое в $(\widehat{x}, \widehat{u})$, т. е. непрерывно в некоторой окрестности $(\widehat{x}, \widehat{u})$, дифференцируемо по $x$ в точке $(\widehat{x}, \widehat{u})$ и для любого $\varepsilon>0$ найдутся такие окрестности $V(\varepsilon)$ и $U(\varepsilon)$ точек $\widehat{x}$ и $\widehat{u}$, что для всех $x, x^{\prime} \in V(\varepsilon)$ и $u \in U(\varepsilon)$ справедливо неравенство

$$
\left\|\Phi(x, u)-\Phi\left(x^{\prime}, u\right)-\Phi_{x}(\widehat{x}, \widehat{u})\left(x-x^{\prime}\right)\right\|_{Z} \leqslant \varepsilon\left\|x-x^{\prime}\right\|_{X} \cdot{ }^{3}
$$

Функиия Лагранжа задачи (Р) имеет вид

$$
\mathscr{L}((x, u), \bar{\lambda})=\lambda_{0} f_{0}(x, u)+\left\langle\lambda_{1}, F(x, u)\right\rangle+\left\langle\lambda_{2}, G(x)\right\rangle,
$$

где $\bar{\lambda}=\left(\lambda_{0}, \lambda_{1}, \lambda_{2}\right) \in \mathbb{R} \times Y_{1}^{*} \times Y_{2}^{*}-$ набор множителей Лагранжа $\left(Y_{i}^{*}-\right.$ сопряженное пространство к $Y_{i},\left\langle\lambda_{i}, y_{i}\right\rangle$ - значение линейного функционала $\lambda_{i} \in Y_{i}^{*}$ на элементе $\left.y_{i} \in Y_{i}, i=1,2\right)$.

Напомним, что если $Q$ - подмножество нормированного пространства $Y$ и $\widehat{y} \in Q$, то множество $N_{Q}(\widehat{y})=\left\{y^{*} \in Y^{*} \mid\left\langle y^{*}, y-\widehat{y}\right\rangle \leqslant 0 \forall y \in Q\right\}$ называется нормальным конусом $к$ множеству $Q$ в точке $\widehat{y}$.

ОсновНАЯ тЕОРемА (принцип Лагранжа для гладко-аппроксимативно-вы-

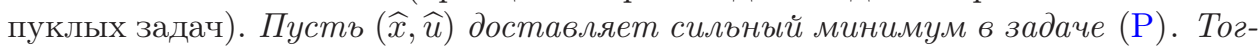
да, если $X, Y_{1}$ и $Y_{2}$ - банаховы пространства, отображение $\left(f_{0}, F\right): V \times \mathscr{U} \rightarrow$ $\mathbb{R} \times Y$ гладко-аппроксимативно-выпукло в точке $(\widehat{x}, \widehat{u})$, отображение $G$ дифференцируемо в $\widehat{x}, \operatorname{codim} \operatorname{Im} F_{x}(\widehat{x}, \widehat{u})<\infty$, множество $Q$ выпукло, замкнуто $u$ int $Q \neq \varnothing$, то найдется такой ненулевой набор множителей Лагранжа $\bar{\lambda}=\left(\lambda_{0}, \lambda_{1}, \lambda_{2}\right) \in \mathbb{R}_{+} \times Y_{1}^{*} \times N_{Q}(G(\widehat{x}))$, что выполняются условие стационарности по $x$ :

$$
\mathscr{L}_{x}((\widehat{x}, \widehat{u}), \bar{\lambda})=0 \Leftrightarrow \lambda_{0} f_{0 x}(\widehat{x}, \widehat{u})+\left(F_{x}(\widehat{x}, \widehat{u})\right)^{*} \lambda_{1}+\left(G^{\prime}(\widehat{x})\right)^{*} \lambda_{2}=0
$$

$\left(\Lambda^{*}\right.$ - сопряженный оператор $\left.\kappa \Lambda\right)$ и условие минимума по $и$ :

$$
\begin{array}{rl}
\min _{u \in \mathscr{U}} & \mathscr{L}((\widehat{x}, u), \bar{\lambda})=\mathscr{L}((\widehat{x}, \widehat{u}), \bar{\lambda}) \\
& \Leftrightarrow \lambda_{0} f_{0}(\widehat{x}, u)+\left\langle\lambda_{1}, F(\widehat{x}, u)\right\rangle \geqslant \lambda_{0} f_{0}(\widehat{x}, \widehat{u})+\left\langle\lambda_{1}, F(\widehat{x}, \widehat{u})\right\rangle \forall u \in \mathscr{U} .
\end{array}
$$

\footnotetext{
${ }^{2}$ Из последнего условия легко следует, что замыкание образа множества $\mathscr{U}$ при отображении $u \mapsto \Phi(\widehat{x}, u)$ выпукло.

${ }^{3}$ Если отображение $\Phi$ не зависит от $u$, то это условие означает строгую дифференцируемость $\Phi: V \rightarrow Z$ в точке $\widehat{x}$.
} 
Краткая формулировка этой теоремы такова: если отображения в задаче $(\mathrm{P})$ гладко-аппроксимативно-выпуклы (в этом состоят условия гладкости и выпуклости), то необходимые условия минимума, т. е. соотношения (I), (II) и включение $\lambda_{2} \in N_{Q}(G(\widehat{x}))$, соответствуют принципу Лагранжа. Действительно, первое есть условие стационарности в точке $\widehat{x}$ в гладкой задаче $\mathscr{L}((x, \widehat{u}), \bar{\lambda}) \rightarrow \min$, $x \in X$; второе есть условие минимума в аппроксимативно-выпуклой задаче $\mathscr{L}((\widehat{x}, u), \bar{\lambda}) \rightarrow \min , u \in \mathscr{U}$. Введя новую переменную $y$, включение $G(x) \in Q$ можно переписать так: $G(x)-y=0, y \in Q$. Последнее слагаемое в функции Лагранжа в этом случае будет иметь вид $\left\langle\lambda_{2}, G(x)-y\right\rangle$, и тогда включение $\lambda_{2} \in N_{Q}(G(\widehat{x}))$ есть условие минимума в выпуклой задаче $\left\langle\lambda_{2}, G(x)-y\right\rangle \rightarrow \min$, $y \in Q$.

Основная теорема является следствием теоремы о разрешимости системы, состоящей из уравнения и включения: $\Phi(x, u)=z, G(x) \in Q$ и стандартных теорем отделимости. Удобно здесь привести формулировку теоремы о разрешимости и предложение об иной трактовке условий регулярности в этой теореме.

Теорема (о разрешимости). Пусть $X, Z_{1}$ и $Z_{2}$ - банаховы пространства, $\mathscr{U}$ - топологическое пространство, $V$ - окрестность точки $\widehat{x} \in X, \widehat{u} \in \mathscr{U}$, $Q \subset Z_{2}, \Phi: V \times \mathscr{U} \rightarrow Z_{1} u G: V \rightarrow Z_{2}$. Тогда, если

1) $\Phi(\widehat{x}, \widehat{u})=0, G(\widehat{x}) \in Q$;

2) отображение $\Phi$ гладко-аппроксимативно-выпукло в точке $(\widehat{x}, \widehat{u})$;

3) отображение $G$ дифферениируемо в точке $\widehat{x}$;

4) множество $Q$ выпукло, замкнуто $u$ int $Q \neq \varnothing$;

5) выполнень условия регулярности: $\operatorname{codim} \operatorname{Im} \Phi_{x}(\widehat{x}, \widehat{u})<\infty u$

$$
0 \in \operatorname{int}\left(\operatorname{Im}\left(\Phi_{x}(\widehat{x}, \widehat{u}), G^{\prime}(\widehat{x})\right)+(\operatorname{co} \Phi(\widehat{x}, \mathscr{U}), G(\widehat{x})-Q)\right),{ }^{4}
$$

то существуют такие окрестности $V_{0} u W_{0}$ точек $\widehat{x}$ и нуля в $Z_{1}$ и константа $K>0$, что для каждого $z \in W_{0}$ найдутся $x(z) \in V_{0} u u(z) \in \mathscr{U}$, для которьх $\Phi(x(z), u(z))=z, G(x(z)) \in Q u\|x(z)-\widehat{x}\|_{X} \leqslant K\|z\|_{Z_{1}}$.

ПредлОжЕНИЕ 1 (об условиях регулярности). Условия регулярности в теореме о разрешимости равносильны условиям: $\operatorname{codim} \operatorname{Im} \Phi_{x}(\widehat{x}, \widehat{u})<\infty$ и не существует линейных функиионалов $\lambda_{1} \in Z_{1}^{*}$ и $\lambda_{2} \in N_{Q}(G(\widehat{x}))$, не равных одновременно нулю, таких, что

$$
\begin{gathered}
\left(\Phi_{x}(\widehat{x}, \widehat{u})\right)^{*} \lambda_{1}+\left(G^{\prime}(\widehat{x})\right)^{*} \lambda_{2}=0, \\
\left\langle\lambda_{1}, \Phi(\widehat{x}, u)\right\rangle \geqslant\left\langle\lambda_{1}, \Phi(\widehat{x}, \widehat{u})\right\rangle=0 \quad \forall u \in \mathscr{U} .
\end{gathered}
$$

\section{2. Следствия}

В этом разделе показывается, что необходимые условия минимума для классов задач, описанных выше, являются следствиями принципа Лагранжа для гладко-аппроксимативно-выпуклых задач.

\footnotetext{
${ }^{4}$ Если $X, Z_{1}$ и $Z_{2}$ - векторные пространства и $A_{i}: X \rightarrow Z_{i}, i=1,2,-$ линейные операторы, то линейный оператор $\left(A_{1}, A_{2}\right): X \rightarrow Z_{1} \times Z_{2}$ действует по правилу $\left(A_{1}, A_{2}\right) x=\left(A_{1} x, A_{2} x\right)$.
} 
2.1. Математическое программирование. Пусть $X$ и $Y$ - нормированные пространства, $V$ - открытое подмножество $X, f_{i}: V \rightarrow \mathbb{R}, i=0,1, \ldots, m$, и $F: V \rightarrow Y$. Задачу

$$
f_{0}(x) \rightarrow \min , \quad f_{i}(x) \leqslant 0, \quad 1 \leqslant i \leqslant m, \quad F(x)=0
$$

называют задачей с ограничениями типа равенств и неравенств (или задачей математического программирования). Функция Лагранжа задачи $\left(\mathrm{P}_{1}\right)$ имеет вид:

$$
\mathscr{L}(x, \bar{\lambda})=\sum_{i=0}^{m} \lambda_{i} f_{i}(x)+\langle\lambda, F(x)\rangle,
$$

где $\bar{\lambda}=\left(\lambda_{0}, \lambda_{1}, \ldots, \lambda_{m}, \lambda\right) \in\left(\mathbb{R}^{m+1}\right)^{*} \times Y^{*}-$ набор множителей Лагранжа. (Напомним, что сопряженное пространство к $\mathbb{R}^{n}$, обозначаемое $\left(\mathbb{R}^{n}\right)^{*}$, отождествляется с пространством вектор-строк. Если $a=\left(a_{1}, \ldots, a_{n}\right) \in\left(\mathbb{R}^{n}\right)^{*}$ и $x=\left(x_{1}, \ldots, x_{n}\right)^{T} \in \mathbb{R}^{n}(T-$ транспонирование $)$, то $\langle a, x\rangle=\sum_{i=1}^{n} a_{i} x_{i}$ - значение
линейного функционала $a$ на элементе $x$. $)$

СледствиЕ 1 (принцип Лагранжа для гладких задач с равенствами и неравенствами). Пусть $\widehat{x}$ - локальный минимум в задаче $\left(\mathrm{P}_{1}\right)$. Тогда, если $X$ и $Y$ банаховы пространства, функиия $f_{0}$ и отображение $F$ строго дифферениируемъ в $\widehat{x} \in V$, функиии $f_{i}, i=1, \ldots, m$, дифферениируемы в $\widehat{x} u \operatorname{Im} F^{\prime}(\widehat{x})$ замкнутое подпространство, то найдется такой ненулевой набор множителей Лагранжа $\bar{\lambda}=\left(\lambda_{0}, \lambda_{1}, \ldots, \lambda_{m}, \lambda\right)$, что выполняются следуюшие условия:

(а) $\mathscr{L}_{x}(\widehat{x}, \bar{\lambda})=0$ тогда и только тогда, когда $\sum_{i=0}^{m} \lambda_{i} f_{i}^{\prime}(\widehat{x})+\left(F^{\prime}(\widehat{x})\right)^{*} \lambda=0$ (условие стационарности);

(b) $\lambda_{i} \geqslant 0, i=0,1, \ldots, m$ (условия неотрицательности);

(c) $\lambda_{i} f_{i}(\widehat{x})=0, i=1, \ldots, m$ (условия дополняющей нежесткости).

ДокАЗАтельство. Сопоставим задаче $\left(\mathrm{P}_{1}\right)$ задачу в форме общей постановки $(\mathrm{P})$, где $Y_{1}=Y, Y_{2}=\mathbb{R}^{m}$, топологическое пространство состоит из одной точки (иначе говоря, $f_{0}$ и $F$ не зависят от $u$ ), отображение $G: V \rightarrow Y_{2}$ задается формулой $G(x)=\left(f_{1}(x), \ldots, f_{m}(x)\right)^{T}$ и $Q=\left\{y=\left(y_{1}, \ldots, y_{m}\right)^{T} \in \mathbb{R}^{m}\right.$ । $\left.y_{i} \leqslant 0,1 \leqslant i \leqslant m\right\}$. Гладкость отображения $\left(f_{0}, F\right)$ следует из строгой дифференцируемости $f_{0}$ и $F$. Пусть $\operatorname{Im} F^{\prime}(\widehat{x})=Y$, тогда $\operatorname{codim} \operatorname{Im} F^{\prime}(\widehat{x})=0$ и, тем самым, выполнены все предположения основной теоремы. Условие стационарности (I) есть утверждение (a). Условие дополняющей нежесткости можно считать выполненным всегда (отбросив те ограничения, где $f_{i}(\widehat{x})<0$ ). Включение $\left(\lambda_{1}, \ldots, \lambda_{m}\right) \in N_{Q}(G(\widehat{x}))$ означает, что $\sum_{i=1}^{m} \lambda_{i} y_{i} \leqslant \sum_{i=1}^{m} \lambda_{i} f_{i}(\widehat{x})$ для всех $y=\left(y_{1}, \ldots, y_{m}\right)^{T} \in Q$. Так как $\sum_{i=1}^{m} \lambda_{i} f_{i}(\widehat{x})=0$ в силу $($ с $)$, то $\lambda_{i} \geqslant 0, i=1, \ldots, m$. Неотрицательность $\lambda_{0}$ следует из основной теоремы.

Если $\operatorname{Im} F^{\prime}(\widehat{x}) \neq Y$, то у замкнутого подпространства $\operatorname{Im} F^{\prime}(\widehat{x})$ есть нетривиальный аннулятор $\lambda \in Y^{*}$ и поэтому для набора $\bar{\lambda}=(0, \ldots, 0, \lambda)$ выполняется условие (а) и очевидным образом - остальные условия. Следствие доказано. 
ЗАмечАниЕ 1. Необходимые условия минимума в общей задаче Лагранжа классического вариационного исчисления являются непосредственным следствием доказанного утверждения (см., например, [13]).

2.2. Выпуклое программирование. Пусть $Z$ - нормированное пространство, $A$ - выпуклое подмножество $Z$ и $g_{i}: Z \rightarrow \mathbb{R}, i=0,1, \ldots, m,-$ выпуклые функции. Задачу

$$
g_{0}(z) \rightarrow \min , \quad g_{i}(z) \leqslant 0, \quad 1 \leqslant i \leqslant m, \quad z \in A,
$$

называют выпуклой задачей или задачей выпуклого программирования. Функция Лагранжа данной задачи имеет вид

$$
\mathscr{L}(z, \bar{\lambda})=\sum_{i=0}^{m} \lambda_{i} g_{i}(z)
$$

где $\bar{\lambda}=\left(\lambda_{0}, \lambda_{1}, \ldots, \lambda_{m}\right) \in\left(\mathbb{R}^{m+1}\right)^{*}$ - набор множителей Лагранжа.

СлЕдствиЕ 2 (теорема Каруша-Куна-Таккера - принцип Лагранжа для выпуклых задач). Если $\widehat{z}$ - минимум в задаче $\left(\mathrm{P}_{2}\right)$, то найдется такой ненулевой набор множителей Лагранжа $\bar{\lambda}=\left(\lambda_{0}, \lambda_{1}, \ldots, \lambda_{m}\right)$, что выполняются следующие условия:

(a) $\min _{z \in A} \mathscr{L}(z, \bar{\lambda})=\mathscr{L}(\widehat{z}, \bar{\lambda})$ (условие минимума);

(b) $\lambda_{i} \geqslant 0, i=0,1, \ldots, m$ (условия неотрицательности);

(c) $\lambda_{i} g_{i}(\widehat{z})=0, i=1, \ldots, m$ (условия дополняющей нежесткости).

Если существуют допустимая в $\left(\mathrm{P}_{2}\right)$ точка $\widehat{z}$ и набор $\bar{\lambda}=\left(\lambda_{0}, \lambda_{1}, \ldots, \lambda_{m}\right)$, удовлетворяющие условиям (a), (b) u (c), и при этом $\lambda_{0}>0$, то $\widehat{z}$ - решение задачи $\left(\mathrm{P}_{2}\right)$.

ДокАЗАтельство. Редуцируем задачу $\left(\mathrm{P}_{2}\right)$ к общей задаче $(\mathrm{P})$, полагая $Y_{1}=\mathbb{R}^{m}, \mathscr{U}=A \times \mathbb{R}_{+}^{m+1}$ (элементы $\mathscr{U}$ обозначим $u=(z, v), v=\left(v_{0}, v_{1}, \ldots, v_{m}\right)$ ), $f_{0}(u)=g_{0}(z)+v_{0}, F(u)=\left(g_{1}(z)+v_{1}, \ldots, g_{m}(z)+v_{m}\right)$. Зависимости от $x$ нет, и отсутствуют ограничения типа включения. Легко видеть, что если $\widehat{z}$ является решением задачи $\left(\mathrm{P}_{2}\right)$, то $\widehat{u}=(\widehat{z}, \widehat{v})$, где $\widehat{v}=\left(0,-g_{1}(\widehat{z}), \ldots,-g_{m}(\widehat{z})\right)-$ решение редуцированной задачи. Пусть $\bar{u}=\left(\left(z_{1}, v^{1}\right), \ldots,\left(z_{k}, v^{k}\right)\right) \in \mathscr{U}^{k}$; микс определяем следующим образом: $M(\alpha, \delta, \bar{u})=\sum_{i=1}^{k} \alpha_{i}\left(z_{i}, \widetilde{v}^{i}\right)$, где $\widetilde{v}^{i}=v^{i}+\left(g_{0}\left(z_{i}\right)-\right.$ $\left.\sum_{j=1}^{k} \alpha_{j} g_{0}\left(z_{j}\right), \ldots, g_{m}\left(z_{i}\right)-\sum_{j=1}^{k} \alpha_{j} g_{m}\left(z_{j}\right)\right), i=1, \ldots, k$. Просто проверяется, что $M(\alpha, \delta, \bar{u}) \in \mathscr{U}, M\left(e_{i}, \delta, \bar{u}\right)=u_{i}=\left(z_{i}, v^{i}\right), i=1, \ldots, k$, и, очевидно, отображение $\alpha \mapsto M(\alpha, \delta, \bar{u})$ непрерывно. Далее, если $\Phi=\left(f_{0}, F\right)$, то $\Phi(M(\alpha, \delta, \bar{u}))=$ $\sum_{i=1}^{k} \alpha_{i} \Phi\left(u_{i}\right)$. Следовательно, по основной теореме найдется такой ненулевой набор множителей Лагранжа $\left(\lambda_{0}, \lambda_{1}, \ldots, \lambda_{m}\right)$, что для функции Лагранжа $u \mapsto$ $\sum_{i=0}^{m} \lambda_{i}\left(g_{i}(z)+v_{i}\right)$ выполнено условие минимума по $u: \sum_{i=0}^{m} \lambda_{i}\left(g_{i}(z)+v_{i}\right) \geqslant \lambda_{0} g_{0}(\widehat{z})$ для всех $z \in A$ и $\left(v_{0}, v_{1}, \ldots, v_{m}\right) \in \mathbb{R}_{+}^{m+1}$. Полагая $z=\widehat{z}$, получаем, что 
$\lambda_{i} \geqslant 0, i=0,1, \ldots, m$. Далее, полагая $z=\widehat{z}$ и $v=0$, приходим к неравенству $\sum_{i=1}^{m} \lambda_{i} g_{i}(\widehat{z}) \geqslant 0$, откуда, в силу того что $\lambda_{i} g_{i}(\widehat{z}) \leqslant 0, i=1, \ldots, m$, следует (с). Теперь, в условии минимума по $u$ полагая $v=\left(v_{0}, \ldots, v_{m}\right)=0$ и добавляя к правой части нулевое слагаемое $\sum_{i=1}^{m} \lambda_{i} g_{i}(\widehat{z})$, получаем (а). Достаточность проверяется без труда. Следствие доказано.

2.3. Ляпуновские задачи. Пусть $\Delta$ - промежуток числовой прямой (конечный или бесконечный), $U$ - непустое подмножество $\mathbb{R}^{r}$ и $\psi_{i}: \Delta \times U \rightarrow \mathbb{R}$, $i=0,1, \ldots, m-$ непрерывные функции. Задачу

$$
\begin{gathered}
g_{0}(u(\cdot))=\int_{\Delta} \psi_{0}(t, u(t)) d t \rightarrow \min , \\
g_{i}(u(\cdot))=\int_{\Delta} \psi_{i}(t, u(t)) d t \leqslant 0, \quad 1 \leqslant i \leqslant m^{\prime}, \\
g_{i}(u(\cdot))=\int_{\Delta} \psi_{i}(t, u(t)) d t=0, \quad m^{\prime}+1 \leqslant i \leqslant m,
\end{gathered}
$$

называют ляпуновской задачей.

Функция Лагранжа задачи $\left(\mathrm{P}_{3}\right)$ имеет вид

$$
\mathscr{L}(u(\cdot), \bar{\lambda})=\sum_{i=0}^{m} \lambda_{i} g_{i}(u(\cdot)),
$$

где $\bar{\lambda}=\left(\lambda_{0}, \lambda_{1}, \ldots, \lambda_{m}\right) \in\left(\mathbb{R}^{m+1}\right)^{*}$ - набор множителей Лагранжа.

Пусть функции $\psi_{i}, i=0,1, \ldots, m$, непрерывны и $\mathscr{V}$ - совокупность таких измеримых отображений $u: \Delta \rightarrow U$, что функции $t \rightarrow \psi_{i}(t, u(t)), i=0,1, \ldots, m$, интегрируемы на $\Delta$. (Измеримость этих функций следует из непрерывности функций $\psi_{i}$.)

СледствиЕ 3 (необходимые и достаточные условия минимума в ляпуновской задаче). Пусть $\widehat{u}(\cdot)$ - минимум в задаче $\left(\mathrm{P}_{3}\right)$. Тогда, если функиии $\psi_{i}: \Delta \times U \rightarrow \mathbb{R}, i=0,1, \ldots, m$, непрерывны, то найдется такой ненулевой набор множителей Лагранжа $\bar{\lambda}=\left(\lambda_{0}, \lambda_{1}, \ldots, \lambda_{m}\right)$, что выполняются следующие условия: (а) $\min _{u \in U} \sum_{i=0}^{m} \lambda_{i} \psi_{i}(t, u)=\sum_{i=0}^{m} \lambda_{i} \psi_{i}(t, \widehat{u}(t))$ (условие минимума для почти

(b) $\lambda_{i} \geqslant 0, i=0,1, \ldots, m$ (условия неотрицательности);

(c) $\lambda_{i} g_{i}(\widehat{u}(\cdot))=0, i=1, \ldots, m$ (условия дополняющей нежесткости).

Если существуют допустимая в $\left(\mathrm{P}_{3}\right)$ функиия $\widehat{u}(\cdot)$ и набор множителей Лагранжа $\bar{\lambda}=\left(\lambda_{0}, \lambda_{1}, \ldots, \lambda_{m}\right)$, удовлетворяющие условиям (a), (b) u (c), и при этом $\lambda_{0}>0$, то $\widehat{u}(\cdot)$ - решение задачи $\left(\mathrm{P}_{3}\right)$.

ДокАЗАтельство. Редуцируем задачу $\left(\mathrm{P}_{3}\right)$ к задаче $(\mathrm{P})$, полагая $Y=\mathbb{R}^{m}$, $\mathscr{U}=\mathscr{V} \times \mathbb{R}_{+}^{m+1}$ (т.е. $u \in \mathscr{U}$ тогда и только тогда, когда $u=(u(\cdot), v)$, где $u(\cdot) \in \mathscr{V}$ и $\left.v=\left(v_{0}, v_{1}, \ldots, v_{m}\right) \in \mathbb{R}_{+}^{m+1}\right), f_{0}(u)=g_{0}(u(\cdot))+v_{0}$ и $F(u)=$ 
$\left(g_{1}(u(\cdot))+v_{1}, \ldots, g_{m^{\prime}}(u(\cdot))+v_{m^{\prime}}, g_{m^{\prime}+1}(u(\cdot)), \ldots, g_{m}(u(\cdot))\right)$. Зависимости от $x$ нет и ограничения типа включения отсутствуют. Легко видеть, что $\widehat{u}(\cdot)-$ решение задачи $\left(\mathrm{P}_{3}\right)$ в том и только том случае, когда пара $\widehat{u}=(\widehat{u}(\cdot), \widehat{v})$, где $\widehat{v}=\left(0,-g_{1}(\widehat{u}(\cdot)), \ldots,-g_{m^{\prime}}(\widehat{u}(\cdot)), 0, \ldots, 0\right)$, есть решение редуцированной задачи. Образ отображения $u(\cdot) \mapsto\left(g_{0}(u(\cdot)), g_{1}(u(\cdot)), \ldots, g_{m}(u(\cdot))\right), u(\cdot) \in \mathscr{V}$, как следует из теоремы Ляпунова о векторных мерах (см. [9]), есть выпуклое множество. Отсюда вытекает, что образ отображения $u \mapsto \Phi(u)=\left(f_{0}(u), F(u)\right)$ - также выпуклое множество. Тогда, если $\bar{u}=\left(\left(u_{1}(\cdot), v^{1}\right), \ldots,\left(u_{k}(\cdot), v^{k}\right)\right) \in \mathscr{U}^{k}$, то микс определяем так: $M(\alpha, \delta, \bar{u})=\sum_{i=1}^{k} \alpha_{i}\left(u_{i}(\cdot), v^{i}\right)$ и нужные его свойства проверяются очевидным образом. Согласно основной теореме найдется такой ненулевой набор множителей Лагранжа $\left(\lambda_{0}, \lambda_{1}, \ldots, \lambda_{m}\right)$, что для функции Лагранжа $u(\cdot) \mapsto \sum_{i=0}^{m^{\prime}} \lambda_{i}\left(\int_{\Delta} \psi_{i}(t, u(t)) d t+v_{i}\right)+\sum_{i=m^{\prime}+1}^{m} \lambda_{i} \int_{\Delta} \psi_{i}(t, u(t)) d t$ справедливы соотношения (I) и (II). Откуда, так же как и в следствии 2, получаем, что справедливы условия неотрицательности, дополняющей нежесткости и условие минимума:

$$
\min _{u(\cdot) \in \mathscr{V}} \int_{\Delta}\left(\sum_{i=0}^{m} \lambda_{i} \psi_{i}(t, u(t))\right) d t=\int_{\Delta}\left(\sum_{i=0}^{m} \lambda_{i} \psi_{i}(t, \widehat{u}(t))\right) d t .
$$

Покажем, что это условие равносильно (а). Действительно, если выполнено (а), то, очевидно, выполнено (2.1). Пусть справедливо (2.1). Докажем, что условие минимума в следствии 3 имеет место для всех точек множества $A \subset\left[t_{0}, t_{1}\right]$ полной меры, на котором функция $\widehat{u}(\cdot)$ аппроксимативно непрерывна ${ }^{5}$. Предположим, что это не так. Тогда найдутся такие $\tau \in A$ и $v \in U$, что имеет место неравенство $\sum_{i=0}^{m} \lambda_{i} \psi_{i}(\tau, v)<\sum_{i=0}^{m} \lambda_{i} \psi_{i}(\tau, \widehat{u}(\tau))$. Функции слева и справа непрерывны в $\tau$ вдоль некоторого множества $A_{0}$ такого, что $\operatorname{mes}\left(A_{0} \cap[\tau-h, \tau+h]\right)>0$ для всех достаточно малых $h$, и поэтому неравенство сохраняется на некотором множестве $B$ положительной меры. Тогда, если взять функцию $u(\cdot)$ такую, что $u(t)=v$ при $t \in B$ и $u(t)=\widehat{u}(t)$ при $t \notin B$, то она, очевидно, принадлежит $\mathscr{U}$ и мы приходим к противоречию с (2.1). Следствие 3 доказано.

2.4. Задачи оптимального управления. Пусть $\left[t_{0}, t_{1}\right]$ - отрезок прямой, $\mathscr{O}$ - открытое подмножество $\mathbb{R} \times \mathbb{R}^{n}, W$ - проекция $\mathscr{O}$ на $\mathbb{R}^{n}, U$ - непустое подмножество $\mathbb{R}^{r}$, заданы функции $f: \mathscr{O} \times \operatorname{cl} U \rightarrow \mathbb{R}, g_{i}: \mathscr{O} \rightarrow \mathbb{R}, i=1, \ldots, m$, и отображения $\varphi: \mathscr{O} \times \operatorname{cl} U \rightarrow \mathbb{R}^{n}$ и $h_{i}: W \rightarrow \mathbb{R}^{s_{i}}, i=0,1$. Рассматривается следующая задача оптимального управления с фазовыми ограничениями:

$$
J(x(\cdot), u(\cdot))=\int_{t_{0}}^{t_{1}} f(t, x(t), u(t)) d t \rightarrow \min , \quad \dot{x}=\varphi(t, x, u),
$$

$$
h_{i}\left(x\left(t_{i}\right)\right)=0, \quad i=0,1, \quad u(t) \in U, \quad g_{i}(t, x(t)) \leqslant 0, \quad i=1, \ldots, m .
$$

\footnotetext{
${ }^{5}$ Точка $\tau \in\left[t_{0}, t_{1}\right]$ есть точка аппроксимативной непрерывности $\widehat{u}(\cdot)$, если существует такое измеримое множество $A \subset\left[t_{0}, t_{1}\right]$, что $\lim _{h \rightarrow 0} \operatorname{mes}(A \cap[\tau-h, \tau+h]) /(2 h)=1$ и функция $\widehat{u}(\cdot)$ непрерывна в $\tau$ вдоль $A$.
} 
Обозначим через $A C\left(\left[t_{0}, t_{1}\right], \mathbb{R}^{n}\right)$ совокупность абсолютно непрерывных вектор-функций на отрезке $\left[t_{0}, t_{1}\right]$. Пару $(x(\cdot), u(\cdot)) \in A C\left(\left[t_{0}, t_{1}\right], \mathbb{R}^{n}\right) \times L_{\infty}\left(\left[t_{0}, t_{1}\right], \mathbb{R}^{r}\right)$ назовем допустимым (управляемым) процессом в задаче $\left(\mathrm{P}_{4}\right)$, если $\Gamma(x(\cdot))=$ $\left\{(t, x(t)) \mid t \in\left[t_{0}, t_{1}\right]\right\} \subset \mathscr{O}$, равенство $\dot{x}(t)=\varphi(t, x(t), u(t))$ и включение $u(t) \in U$ выполняются для почти всех $t \in\left[t_{0}, t_{1}\right]$, неравенства $g_{i}(t, x(t)) \leqslant 0, i=1, \ldots, m$, выполняются для всех $t \in\left[t_{0}, t_{1}\right]$ и $h_{i}\left(x\left(t_{i}\right)\right)=0, i=0,1$.

Допустимый процесс $(\widehat{x}(\cdot), \widehat{u}(\cdot))$ называется оптимальным процессом (или сильным минимумом), если существует такое $\varepsilon>0$, что для всякого допустимого процесса $(x(\cdot), u(\cdot))$, для которого $\|x(\cdot)-\widehat{x}(\cdot)\|_{C\left(\left[t_{0}, t_{1}\right], \mathbb{R}^{n}\right)}<\varepsilon$, выполняется неравенство $J(x(\cdot), u(\cdot)) \geqslant J(\widehat{x}(\cdot), \widehat{u}(\cdot))$.

Функция

$$
H\left(t, x, u, \lambda_{0}, p(\cdot)\right)=(p(t), \varphi(t, x, u))-\lambda_{0} f(t, x, u),
$$

где $\lambda_{0} \in \mathbb{R}$ и $p(\cdot):\left[t_{0}, t_{1}\right] \rightarrow\left(\mathbb{R}^{n}\right)^{*}$, называется функиией Понтрягина задачи $\left(\mathrm{P}_{4}\right)$.

СледСтвиЕ 4 (необходимые условия минимума в задаче оптимального управления - принцип максимума Понтрягина). Пусть $(\widehat{x}(\cdot), \widehat{u}(\cdot))$ - оптимальныи процесс в задаче $\left(\mathrm{P}_{4}\right)$. Тогда, если функция $f$, отображсение $\varphi$ и функции $g_{i}$, $i=1, \ldots, m$, непрерывны вместе со своими частными производными по $x$ соответственно на $\mathscr{O} \times \operatorname{cl} U$ u $\mathscr{O}$, а отображения $h_{i}, i=0,1$, непрерывно дифберениируемы на $W$, то найдутся, не равные одновременно нулю, число $\lambda_{0} \geqslant 0$, векторы $l_{i} \in\left(\mathbb{R}^{s_{i}}\right)^{*}, i=0,1$, функция $p(\cdot):\left[t_{0}, t_{1}\right] \rightarrow\left(\mathbb{R}^{n}\right)^{*}$ ограниченной вариации, непрерывная справа на $\left(t_{0}, t_{1}\right)$, неотрицательная борелевская векторная мера $d \mu=\left(d \mu_{1}, \ldots, d \mu_{n}\right)$ на $\left[t_{0}, t_{1}\right]$, где $d \mu_{i}$ сосредоточена на множестве $T_{i}=\left\{t \in\left[t_{0}, t_{1}\right] \mid g_{i}(t, \widehat{x}(t))=0\right\}, i=1, \ldots, m$, такие, что для всех $t \in\left[t_{0}, t_{1}\right]$ выполняются равенство

$$
\begin{aligned}
p(t)= & -\widehat{h}_{1}^{\prime *} l_{1}+\int_{t}^{t_{1}} H_{x}\left(\tau, \widehat{x}(\tau), \widehat{u}(\tau), \lambda_{0}, p(\tau)\right) d \tau \\
& -\sum_{i=1}^{m} \int_{t}^{t_{1}} g_{i x}(\tau, \widehat{x}(\tau)) d \mu_{i}, \quad p\left(t_{0}\right)=\widehat{h}_{0}^{\prime *} l_{0},
\end{aligned}
$$

и для почти всех $t \in\left[t_{0}, t_{1}\right]$ соотношение

$$
\max _{u \in U} H\left(t, \widehat{x}(t), u, \lambda_{0}, p(t)\right)=H\left(t, \widehat{x}(t), \widehat{u}(t), \lambda_{0}, p(t)\right) .
$$

ДокАЗАТЕльство. Запишем задачу $\left(\mathrm{P}_{4}\right)$ в форме задачи $(\mathrm{P})$, где

$$
\begin{gathered}
X=C\left(\left[t_{0}, t_{1}\right], \mathbb{R}^{n}\right), \quad Y_{1}=C\left(\left[t_{0}, t_{1}\right], \mathbb{R}^{n}\right) \times \mathbb{R}^{s_{0}} \times \mathbb{R}^{s_{1}}, \quad Y_{2}=C\left(\left[t_{0}, t_{1}\right], \mathbb{R}^{m}\right), \\
V=\left\{x(\cdot) \in C\left(\left[t_{0}, t_{1}\right], \mathbb{R}^{n}\right) \mid \Gamma(x(\cdot)) \subset \mathscr{O}\right\}, \\
\mathscr{U}=\left\{u(\cdot) \in L_{\infty}\left(\left[t_{0}, t_{1}\right], \mathbb{R}^{r}\right) \mid u(t) \in U \text { для почти всех } t \in\left[t_{0}, t_{1}\right]\right\}
\end{gathered}
$$

$\left(\mathscr{U}\right.$ рассматривается в топологии $\left.L_{1}\left(\left[t_{0}, t_{1}\right], \mathbb{R}^{r}\right)\right), f_{0}: V \times \mathscr{U} \rightarrow \mathbb{R}, f_{0}(x(\cdot), u(\cdot))=$ $J(x(\cdot), u(\cdot)), Q=\left\{y(\cdot) \in Y_{2} \mid y(t) \leqslant 0 \forall t \in\left[t_{0}, t_{1}\right]\right\}$, отображения $F: V \times \mathscr{U} \rightarrow Y_{1}$ и $G: V \rightarrow Y_{2}$ определены соответственно формулами

$$
F(x(\cdot), u(\cdot))(t)=\left(x(t)-x\left(t_{0}\right)-\int_{t_{0}}^{t} \varphi(\tau, x(\tau), u(\tau)) d \tau, h_{0}\left(x\left(t_{0}\right)\right), h_{1}\left(x\left(t_{1}\right)\right)\right)
$$


для всех $t \in\left[t_{0}, t_{1}\right]$ и

$$
G(x(\cdot))(\cdot)=\left(g_{1}(\cdot, x(\cdot)), \ldots, g_{m}(\cdot, x(\cdot))\right)
$$

Ясно, что если $(\widehat{x}(\cdot), \widehat{u}(\cdot))$ - оптимальный процесс в $\left(\mathrm{P}_{4}\right)$, то $(\widehat{x}(\cdot), \widehat{u}(\cdot))$ - сильный минимум в соответствующей задаче $(\mathrm{P})$.

Проверим выполнение условий основной теоремы. Покажем, что отображение $\Phi=\left(f_{0}, F\right)$ гладко-аппроксимативно-выпукло в точке $(\widehat{x}(\cdot), \widehat{u}(\cdot))$. Начнем с построения микса. Пусть $\bar{u}(\cdot)=\left(u_{1}(\cdot), \ldots, u_{m}(\cdot)\right) \in \mathscr{U}^{m}, \alpha=\left(\alpha_{1}, \ldots, \alpha_{m}\right) \in$ $\Sigma^{m}$ и $\delta>0$. Можно считать, что $0<\delta<t_{1}-t_{0}$. Разобьем отрезок $\left[t_{0}, t_{1}\right]$ на подотрезки $\Delta$ длины не больше $\delta(|\Delta| \leqslant \delta)$. Затем каждый из таких подотрезков разбиваем на подподотрезки $\Delta_{1}, \ldots, \Delta_{m}$ соответственно длин $\alpha_{1}|\Delta|, \ldots, \alpha_{m}|\Delta|$ и полагаем $M(\alpha, \delta, \bar{u}(\cdot))(t)=u_{i}(t)$, если $t \in \Delta_{i}, 1 \leqslant i \leqslant m$. Легко проверить, что $M\left(e_{i}, \delta, \bar{u}(\cdot)\right)(\cdot)=u_{i}(\cdot), 1 \leqslant i \leqslant m$, и отображение $\alpha \mapsto M(\alpha, \delta, \bar{u}(\cdot))(\cdot)$ непрерывно равномерно по $\delta$.

Остальные свойства (в определении аппроксимативной выпуклости и гладкости) суть свойства интегрального отображения, и поэтому мы проверим их лишь для отображения $\mathscr{F}: V \times \mathscr{U} \rightarrow C\left(\left[t_{0}, t_{1}\right], \mathbb{R}^{n}\right)$, задаваемого формулой $\left(t \in\left[t_{0}, t_{1}\right]\right)$

$$
\mathscr{F}(x(\cdot), u(\cdot))(t)=\int_{t_{0}}^{t} \varphi(\tau, x(\tau), u(\tau)) d \tau
$$

В общем случае рассуждения совершенно аналогичны.

Покажем, что $\mathscr{F}(\widehat{x}(\cdot), M(\alpha, \delta, \bar{u}(\cdot)))(\cdot) \rightarrow \sum_{i=1}^{m} \alpha_{i} \mathscr{F}\left(\widehat{x}(\cdot), u_{i}(\cdot)\right)(\cdot)$ в $C\left(\left[t_{0}, t_{1}\right], \mathbb{R}^{n}\right)$ при $\delta \rightarrow 0$ равномерно на $\Sigma^{m}$. Пусть $0<\varepsilon<t_{1}-t_{0}$. Из $C$-свойства Лузина и регулярности меры Лебега следует существование такого замкнутого множества $A=A(\varepsilon) \subset\left[t_{0}, t_{1}\right]$, что mes $A>\left(t_{1}-t_{0}\right)-\varepsilon$ и на $A$ функции $\varphi_{i}(\cdot)=$ $\varphi\left(\cdot, \widehat{x}(\cdot), u_{i}(\cdot)\right), 1 \leqslant i \leqslant m$, непрерывны. Кроме того, существуют непрерывные функции $\psi_{i}(\cdot)$ на $\left[t_{0}, t_{1}\right]$ такие, что $\varphi_{i}(\cdot)=\psi_{i}(\cdot)$ на $A$ и $\left\|\psi_{i}(\cdot)\right\|_{C\left(\left[t_{0}, t_{1}\right], \mathbb{R}^{n}\right)} \leqslant$ $\left\|\varphi_{i}(\cdot)\right\|_{L_{\infty}\left(\left[t_{0}, t_{1}\right], \mathbb{R}^{n}\right)}, 1 \leqslant i \leqslant m$. Выберем $\delta>0$ так, чтобы выполнялись неравенства $\left|\psi_{i}(t)-\psi_{i}\left(t^{\prime}\right)\right|<\varepsilon, 1 \leqslant i \leqslant m$, если $\left|t-t^{\prime}\right|<\delta$, и разобьем отрезок $\left[t_{0}, t_{1}\right]$ на подотрезки $\Delta$ длины не больше $\delta$. Теперь несложные вычисления показывают, что для любых $t \in\left[t_{0}, t_{1}\right]$ и $\alpha=\left(\alpha_{1}, \ldots, \alpha_{m}\right) \in \Sigma^{m}$ справедлива оценка $\left|\mathscr{F}(\widehat{x}(\cdot), M(\alpha, \delta, \bar{u}(\cdot)))(t)-\sum_{i=1}^{m} \alpha_{i} \mathscr{F}\left(\widehat{x}(\cdot), u_{i}(\cdot)\right)(t)\right| \leqslant C \varepsilon$ (константа $C$ зависит только от величин $\left.\left\|\varphi_{i}(\cdot)\right\|_{L_{\infty}\left(\left[t_{0}, t_{1}\right], \mathbb{R}^{n}\right)}, 1 \leqslant i \leqslant m\right)$, которая доказывает нужное утверждение.

Проверим условие гладкости (b). Заметим сначала, что для построенного микса $M(\alpha, \delta, \widehat{u}(\cdot))(\cdot)$, очевидно, справедлива оценка

$$
|M(\alpha, \delta, \widehat{u}(\cdot))(t)| \leqslant R=\max _{1 \leqslant i \leqslant m}\left\|u_{i}(\cdot)\right\|_{L_{\infty}\left(\left[t_{0}, t_{1}\right], \mathbb{R}^{r}\right)}
$$

для всех $\alpha \in \Sigma^{m}$ и почти всех $t \in\left[t_{0}, t_{1}\right]$. Согласно замечанию 2 в конце п. 2 доказательства теоремы о разрешимости (см. раздел 3 ), достаточно 
в определении гладкости (свойство (b)) рассматривать вместо $\mathscr{U}$ множество $\mathscr{U}_{0}=\left\{u(\cdot) \in L_{\infty}\left(\left[t_{0}, t_{1}\right], \mathbb{R}^{r}\right) \mid u(t) \in U \cap B_{\mathbb{R}^{r}}(0, R)\right.$ для почти всех $\left.t \in\left[t_{0}, t_{1}\right]\right\} .{ }^{6}$

Покажем сначала, что отображение $\mathscr{F}$ непрерывно в окрестности точки $(\widehat{x}(\cdot), \widehat{u}(\cdot))$. Пусть точка $(\bar{u}(\cdot), \bar{x}(\cdot))$ - из этой окрестности. Легко видеть, что существует $\delta_{0}>0$ такое, что компакт $K=\left\{(t, x) \in \mathbb{R}^{n+1}|| x-\bar{x}(t) \mid \leqslant \delta_{0}, t \in\right.$ $\left.\left[t_{0}, t_{1}\right]\right\}$ содержится в $V$. Пусть $\varepsilon>0$. Так как функция $\varphi$ равномерно непрерывна на компакте $K \times\left(\operatorname{cl} U \cap B_{\mathbb{R}^{r}}(0, R)\right)$, то найдется такое $0<\delta_{1} \leqslant \delta_{0}$, что $\left|\varphi\left(t, x_{1}, u_{1}\right)-\varphi\left(t, x_{2}, u_{2}\right)\right|<\varepsilon$ для всех $\left(t, x_{i}, u_{i}\right) \in K \times\left(\operatorname{cl} U \cap B_{\mathbb{R}^{r}}(0, R)\right), i=1,2$, для которых $\left|x_{1}-x_{2}\right|<\delta_{1}$ и $\left|u_{1}-u_{2}\right|<\delta_{1}$.

Пусть $x(\cdot) \in U_{C\left(\Delta, \mathbb{R}^{n}\right)}\left(\bar{x}(\cdot), \delta_{1}\right)$ и $u(\cdot) \in \mathscr{U}_{0} \cap U_{L_{1}\left(\Delta, \mathbb{R}^{r}\right)}\left(\bar{u}(\cdot), \varepsilon \delta_{1}\right)$. Положим $E_{\delta_{1}}=E_{\delta_{1}}(u(\cdot))=\left\{t \in\left[t_{0}, t_{1}\right]|| u(t)-\bar{u}(t) \mid \geqslant \delta_{1}\right\}$. Тогда $\delta_{1} \operatorname{mes} E_{\delta_{1}} \leqslant \int_{E_{\delta_{1}}} \mid u(t)-$ $\bar{u}(t) \mid d t \leqslant\|u(\cdot)-\bar{u}(\cdot)\|_{L_{1}\left(\left[t_{0}, t_{1}\right], \mathbb{R}^{r}\right)}<\varepsilon \delta_{1}$ и, следовательно, mes $E_{\delta_{1}}<\varepsilon$. Для любого $t \in\left[t_{0}, t_{1}\right]$ имеем

$$
\begin{aligned}
|\mathscr{F}(x(\cdot), u(\cdot))(t)-\mathscr{F}(\bar{x}(\cdot), \bar{u}(\cdot))(t)| \\
\leqslant \int_{\left[t_{0}, t_{1}\right] \backslash E_{\delta_{1}}}|\varphi(\tau, x(\tau), u(\tau))-\varphi(\tau, \bar{x}(\tau), \bar{u}(\tau))| d \tau \\
\quad+\int_{E_{\delta_{1}}}|\varphi(\tau, x(\tau), u(\tau))-\varphi(\tau, \bar{x}(\tau), \bar{u}(\tau))| d \tau \\
\quad \leqslant \varepsilon\left(t_{1}-t_{0}\right)+\varepsilon \cdot 2 M_{1},
\end{aligned}
$$

где $M_{1}=\max \left\{|\varphi(t, x, u)| \mid(t, x, u) \in K \times\left(\operatorname{cl} U \cap B_{\mathbb{R}^{r}}(0, R)\right)\right\}$. Отсюда следует непрерывность $\mathscr{F}$ в точке $(\bar{x}(\cdot), \bar{u}(\cdot))$ и, значит, в окрестности $(\widehat{x}(\cdot), \widehat{u}(\cdot))$.

Теперь проверим второе условие в определении гладкости. Пусть $(\bar{x}(\cdot), u(\cdot)) \in$ $V \times \mathscr{U}_{0}$. Так как отображение $\varphi$ непрерывно дифференцируемо по $x$, то стандартные рассуждения показывают, что отображение $\mathscr{F}$ дифференцируемо по $x(\cdot)$ в точке $(\bar{x}(\cdot), u(\cdot))$ и соответствующая производная действует по правилу $\left(t \in\left[t_{0}, t_{1}\right]\right)$

$$
\mathscr{F}_{x(\cdot)}(\bar{x}(\cdot), u(\cdot))[h(\cdot)](t)=\int_{t_{0}}^{t} \varphi_{x}(\tau, \bar{x}(\tau), u(\tau)) h(\tau) d \tau,
$$

где $h(\cdot) \in C\left(\left[t_{0}, t_{1}\right], \mathbb{R}^{n}\right)$. Требуется показать, что для каждого $\varepsilon>0$ найдутся окрестности $V(\varepsilon)$ и $U(\varepsilon)$ точек $\widehat{x}(\cdot)$ и $\widehat{u}(\cdot)$ такие, что для всех $x(\cdot), x^{\prime}(\cdot) \in V(\varepsilon)$ и $u(\cdot) \in U(\varepsilon)$ выполняется неравенство

$$
\begin{aligned}
\| \mathscr{F}(x(\cdot), u(\cdot))(\cdot) & -\mathscr{F}\left(x^{\prime}(\cdot), u(\cdot)\right)(\cdot) \\
& -\mathscr{F}_{x(\cdot)}(\widehat{x}(\cdot), \widehat{u}(\cdot))\left[x(\cdot)-x^{\prime}(\cdot)\right](\cdot) \|_{C\left(\left[t_{0}, t_{1}\right], \mathbb{R}^{n}\right)} \\
& \leqslant \varepsilon\left\|x(\cdot)-x^{\prime}(\cdot)\right\|_{C\left(\left[t_{0}, t_{1}\right], \mathbb{R}^{n}\right)} .
\end{aligned}
$$

Пусть $\varepsilon>0$ и $K$ - то же, что и выше. Функция $\varphi_{x}$ равномерно непрерывна на компакте $K \times\left(\operatorname{cl} U \cap B_{\mathbb{R}^{r}}(0, R)\right)$, и поэтому найдется такое $0<\delta_{1} \leqslant \delta_{0}$, что $\left\|\varphi_{x}\left(t, x_{1}, u_{1}\right)-\varphi_{x}\left(t, x_{2}, u_{2}\right)\right\|<\varepsilon$ для всех $\left(t, x_{i}, u_{i}\right) \in K \times\left(\operatorname{cl} U \cap B_{\mathbb{R}^{r}}(0, R)\right)$, $i=1,2$, для которых $\left|x_{1}-x_{2}\right|<\delta_{1}$ и $\left|u_{1}-u_{2}\right|<\delta_{1}$.

\footnotetext{
6 Здесь и далее $B_{X}(x, r)$ и $U_{X}(x, r)$ обозначают замкнутый и открытый шары в нормированном пространстве $X$ с центром в точке $x$ и радиусом $r$.
} 
Пусть теперь $x(\cdot), x^{\prime}(\cdot) \in U_{C\left(\left[t_{0}, t_{1}\right], \mathbb{R}^{n}\right)}\left(\widehat{x}(\cdot), \delta_{1}\right), \bar{x}(\cdot)=(1-\beta) x(\cdot)+\beta x^{\prime}(\cdot)$, где $0 \leqslant \beta \leqslant 1$ (ясно, что $\left.\bar{x}(\cdot) \in U_{C\left(\left[t_{0}, t_{1}\right], \mathbb{R}^{n}\right)}\left(\widehat{x}(\cdot), \delta_{1}\right)\right), u(\cdot) \in \mathscr{U}_{0} \cap U_{L_{1}\left(\left[t_{0}, t_{1}\right], \mathbb{R}^{r}\right)}\left(\widehat{u}(\cdot), \varepsilon \delta_{1}\right)$, $E_{\delta_{1}}=E_{\delta_{1}}(u(\cdot))$ - то же, что и в рассуждениях выше, $\|h(\cdot)\|_{C\left(\left[t_{0}, t_{1}\right], \mathbb{R}^{n}\right)} \leqslant 1$. Тогда для любого $t \in\left[t_{0}, t_{1}\right]$ имеем

$$
\begin{aligned}
\left|\mathscr{F}_{x(\cdot)}(\bar{x}(\cdot), u(\cdot))[h(\cdot)](t)-\mathscr{F}_{x(\cdot)}(\widehat{x}(\cdot), \widehat{u}(\cdot))[h(\cdot)](t)\right| & \\
= & \left|\int_{t_{0}}^{t}\left(\varphi_{x}(\tau, \bar{x}(\tau), u(\tau))-\varphi_{x}(\tau, \widehat{x}(\tau), \widehat{u}(\tau))\right) h(\tau) d \tau\right| \\
\leqslant & \int_{\left[t_{0}, t_{1}\right] \backslash E_{\delta_{1}}}\left\|\varphi_{x}(\tau, \bar{x}(\tau), u(\tau))-\varphi_{x}(\tau, \widehat{x}(\tau), \widehat{u}(\tau))\right\| d \tau \\
& \quad+\int_{E_{\delta_{1}}}\left\|\varphi_{x}(\tau, \bar{x}(\tau), u(\tau))-\varphi_{x}(\tau, \widehat{x}(\tau), \widehat{u}(\tau))\right\| d \tau \leqslant \varepsilon\left(t_{1}-t_{0}\right)+\varepsilon \cdot 2 M_{2},
\end{aligned}
$$

где $M_{2}=\max \left\{\left\|\varphi_{x}(t, x, u)\right\| \mid(t, x, u) \in K \times\left(\operatorname{cl} U \cap B_{\mathbb{R}^{r}}(0, R)\right)\right\}$. Таким образом, $\left\|\mathscr{F}_{x(\cdot)}(\bar{x}(\cdot), u(\cdot))-\mathscr{F}_{x(\cdot)}(\widehat{x}(\cdot), \widehat{u}(\cdot))\right\| \leqslant \varepsilon\left(t_{1}-t_{0}+2 M_{2}\right)$, и тогда из теоремы о среднем (примененной к отображению $\left.x(\cdot) \mapsto \mathscr{F}(x(\cdot), u(\cdot))(\cdot)-\mathscr{F}_{x(\cdot)}(\widehat{x}(\cdot), \widehat{u}(\cdot)) x(\cdot)\right)$ следует неравенство, равносильное $(2.2)$ (с заменой $\varepsilon$ на $\varepsilon\left(t_{1}-t_{0}+2 M_{2}\right)$ ), где $V(\varepsilon)=U_{C\left(\left[t_{0}, t_{1}\right], \mathbb{R}^{n}\right)}\left(\widehat{x}(\cdot), \delta_{1}\right)$ и $U(\varepsilon)=\mathscr{U}_{0} \cap U_{L_{1}\left(\left[t_{0}, t_{1}\right], \mathbb{R}^{r}\right)}\left(\widehat{u}(\cdot), \varepsilon \delta_{1}\right)$.

Итак, доказано, что отображение $\left(f_{0}, F\right)$ гладко-аппроксимативно-выпукло в $(\widehat{x}(\cdot), \widehat{u}(\cdot))$. Производная по $x(\cdot)$ в точке $(\widehat{x}(\cdot), \widehat{u}(\cdot))$ первой компоненты отображения $F$, действующая в $C\left(\left[t_{0}, t_{1}\right], \mathbb{R}^{n}\right)$, отображает это пространство на подпространство тех функций из $C\left(\left[t_{0}, t_{1}\right], \mathbb{R}^{n}\right)$, которые равны нулю в $t_{0}$ (это простое следствие разрешимости задачи Коши для линейного уравнения). Поскольку это пространство конечной коразмерности, то отсюда нетрудно вывести, что образ производной по $x(\cdot)$ в точке $(\widehat{x}(\cdot), \widehat{u}(\cdot))$ всего отображения $F$ имеет конечную коразмерность. Дифференцируемость отображения $G$ в точке $\widehat{x}(\cdot)$ сразу следует из предположений теоремы, и, наконец, элементарно проверяется, что множество $Q$ выпукло, замкнуто и имеет непустую внутренность.

Все условия основной теоремы выполнены. Следовательно, найдется ненулевой набор множителей Лагранжа $\bar{\lambda}=\left(\lambda_{0}, \lambda_{1}, \lambda_{2}\right) \in \mathbb{R} \times\left(C\left(\left[t_{0}, t_{1}\right], \mathbb{R}^{n}\right) \times \mathbb{R}^{s_{0}} \times\right.$ $\left.\mathbb{R}^{s_{1}}\right)^{*} \times\left(C\left(\left[t_{0}, t_{1}\right], \mathbb{R}^{m}\right)\right)^{*}$, где $\left\langle\lambda_{2}, y(\cdot)-G(\widehat{x}(\cdot))(\cdot)\right\rangle \leqslant 0$ для всех $y(\cdot) \in Q$, такой, что для функции Лагранжа

$$
\begin{aligned}
\mathscr{L}((x(\cdot), u(\cdot)), \bar{\lambda})= & \lambda_{0} \int_{t_{0}}^{t_{1}} f(t, x(t), u(t)) d t+\left\langle\lambda_{1}, F(x(\cdot), u(\cdot))(\cdot)\right\rangle \\
& +\left\langle\lambda_{2}, G(x(\cdot))(\cdot)\right\rangle=\lambda_{0} \int_{t_{0}}^{t_{1}} f(t, x(t), u(t)) d t \\
& +\int_{t_{0}}^{t_{1}}\left(x(t)-x\left(t_{0}\right)-\int_{t_{0}}^{t} \varphi(\tau, x(\tau), u(\tau)) d \tau, d \nu\right) \\
& +\left(l_{0}, h_{0}\left(x\left(t_{0}\right)\right)\right)+\left(l_{1}, h_{1}\left(x\left(t_{1}\right)\right)\right)+\int_{t_{0}}^{t_{1}}(G(x(\cdot))(t), d \mu),
\end{aligned}
$$

где $d \nu=\left(d \nu_{1}, \ldots, d \nu_{m}\right)$ и $d \mu=\left(d \mu_{1}, \ldots, d \mu_{n}\right)$ - борелевские векторные меры на $\left[t_{0}, t_{1}\right]$ (которые отождествляются с функциями ограниченной вариации на $\left(t_{0}, t_{1}\right)$, непрерывными справа), а $l_{i} \in\left(\mathbb{R}^{s_{i}}\right)^{*}, i=0,1$, выполнено условие 
стационарности: $\mathscr{L}_{x(\cdot)}(\widehat{x}(\cdot), \widehat{u}(\cdot), \bar{\lambda})=0$, которое равносильно равенству

$$
\begin{aligned}
& \lambda_{0} \int_{t_{0}}^{t_{1}}\left(\widehat{f}_{x}(t), x(t)\right) d t+\int_{t_{0}}^{t_{1}}\left(x(t)-x\left(t_{0}\right)-\int_{t_{0}}^{t} \widehat{\varphi}_{x}(\tau) x(\tau) d \tau, d \nu\right) \\
& \quad+\left(l_{0}, \widehat{h}_{0}^{\prime} x\left(t_{0}\right)\right)+\left(l_{1}, \widehat{h}_{1}^{\prime} x\left(t_{1}\right)\right)+\sum_{i=1}^{m} \int_{t_{0}}^{t_{1}}\left(g_{i x}(t, \widehat{x}(t)), x(t)\right) d \mu_{i}=0
\end{aligned}
$$

для всех $x(\cdot) \in C\left(\left[t_{0}, t_{1}\right], \mathbb{R}^{n}\right)$. Меняя порядок интегрирования во втором интеграле, после элементарных преобразований это равенство для всех $x(\cdot) \in$ $C\left(\left[t_{0}, t_{1}\right], \mathbb{R}^{n}\right)$ можно записать так:

$$
\begin{aligned}
\int_{t_{0}}^{t_{1}} & \left(x(t), d\left(\int_{t_{0}}^{t} \lambda_{0} \widehat{f}_{x}(\tau) d \tau+\nu(t)+\int_{t_{0}}^{t} \widehat{\varphi}_{x}^{*}(\tau)\left(\int_{\tau}^{t_{1}} d \nu\right) d \tau\right.\right. \\
& \left.\left.\quad-\sum_{i=1}^{m} \int_{t_{0}}^{t} g_{i x}(\tau, \widehat{x}(\tau)) d \mu_{i}+\theta(t)+c\right)\right)+\left(\widehat{h}_{0}^{*} l_{0}-\int_{t_{0}}^{t_{1}} d \nu, x\left(t_{0}\right)\right)=0,
\end{aligned}
$$

где $\theta(t)=0$, если $t_{0} \leqslant t<t_{1}$, и $\theta\left(t_{1}\right)=\widehat{h}_{1}^{\prime *} l_{1}$, а константа $c$ такова, что функция под знаком дифференциала, которую обозначим через $\Psi(\cdot)$, равна нулю в $t_{0}$. Понятно, что $\Psi(\cdot)$ - функция ограниченной вариации, непрерывная справа на $\left(t_{0}, t_{1}\right)$. В силу теоремы Рисса о единственности представления линейного функционала на $C\left(\left[t_{0}, t_{1}\right], \mathbb{R}^{n}\right)$ имеем: $\Psi(t)=\int_{t_{0}}^{t} d \sigma+\nu(t)+\theta(t)+c=0$ для всех $t \in\left[t_{0}, t_{1}\right]$, где $d \sigma-$ сумма соответствующих мер, и $\widehat{h}_{0}^{\prime *} l_{0}=\int_{t_{0}}^{t_{1}} d \nu$. В частности, $\Psi\left(t_{1}\right)=\int_{t_{0}}^{t_{1}} d \sigma+\nu\left(t_{1}\right)+\widehat{h}_{1}^{\prime *} l_{1}+c=0$. Из этих соотношений следует, что $\int_{t}^{t_{1}} d \sigma=\int_{t_{0}}^{t_{1}} d \sigma-\int_{t_{0}}^{t} d \sigma=\nu(t)-\nu\left(t_{1}\right)+\theta(t)-\widehat{h}_{1}^{\prime *} l_{1}$ для всех $t \in\left[t_{0}, t_{1}\right]$. Полагая $p(t)=\int_{t}^{t_{1}} d \nu=\nu\left(t_{1}\right)-\nu(t)$, если $t \in\left[t_{0}, t_{1}\right)$, и $p\left(t_{1}\right)=$ $-\widehat{h}_{1}^{\prime *} l_{1}-\sum_{i=1}^{m} g_{i x}\left(t_{1}, \widehat{x}\left(t_{1}\right)\right) \mu_{i}\left(t_{1}\right)$, приходим к нужному утверждению из следствия 4 .

Покажем, что меры $d \mu_{i}, i=1, \ldots, m$, неотрицательны и сосредоточены соответственно на множествах $T_{i}=\left\{t \in\left[t_{0}, t_{1}\right] \mid \widehat{g}_{i}(t)=0\right\}$. Действительно, из условия $d \mu \in N_{Q}(G(\widehat{x}(\cdot)))$ следует, что $\int_{t_{0}}^{t_{1}}\left(y_{i}(t)-g_{i}(t, \widehat{x}(t))\right) d \mu_{i}(t) \leqslant 0$ для любой неположительной непрерывной функции $y_{i}(\cdot), i=1, \ldots, m$. Фиксируем $1 \leqslant i \leqslant m$. Если $d \mu_{i}$ не является неотрицательной мерой (т. е. существует подмножество $\left[t_{0}, t_{1}\right]$ отрицательной меры), то нетрудно построить непрерывную неположительную функцию $\bar{y}(\cdot)$ такую, что $\int_{t_{0}}^{t_{1}} \bar{y}(t) d \mu_{i}(t)>0$. Но тогда для любого $\alpha \geqslant 0$ должно выполняться неравенство $\int_{t_{0}}^{t_{1}} \alpha \bar{y}(t) d \mu_{i}(t) \leqslant$ $\int_{t_{0}}^{t_{1}} g_{i}(t, \widehat{x}(t)) d \mu_{i}(t)$, что невозможно. Далее, если мера $d \mu_{i}$ не сосредоточена 
на множестве $T_{i}$, то $\int_{t_{0}}^{t_{1}} g_{i}(t, \widehat{x}(t)) d \mu_{i}(t)<0$, что снова невозможно, так как этот интеграл неотрицателен (надо положить $\left.y_{i}(\cdot)=0\right)$.

Условие (II) равносильно тому, что

$$
\begin{array}{r}
\max _{u(\cdot) \in \mathscr{U}} \int_{t_{0}}^{t_{1}}\left(\left(\int_{t_{0}}^{t} \varphi(\tau, \widehat{x}(\tau), u(\tau)) d \tau, d \nu\right)-\lambda_{0} f(t, \widehat{x}(t), u(t)) d t\right) \\
=\int_{t_{0}}^{t_{1}}\left(\left(\int_{t_{0}}^{t} \varphi(\tau, \widehat{x}(\tau), \widehat{u}(\tau)) d \tau, d \nu\right)-\lambda_{0} f(t, \widehat{x}(t), \widehat{u}(t)) d t\right) .
\end{array}
$$

Ho

$$
\begin{aligned}
\int_{t_{0}}^{t_{1}} & \left(\int_{t_{0}}^{t} \varphi(\tau, \widehat{x}(\tau), u(\tau)) d \tau, d \nu\right) \\
& =\int_{t_{0}}^{t_{1}}\left(\int_{\tau}^{t_{1}} d \nu, \varphi(\tau, \widehat{x}(\tau), u(\tau))\right) d \tau=\int_{t_{0}}^{t_{1}}(p(\tau), \varphi(\tau, \widehat{x}(\tau), u(\tau))) d \tau
\end{aligned}
$$

и тогда отсюда и из предыдущего равенства следует, что

$$
\max _{u(\cdot) \in \mathscr{U}} \int_{t_{0}}^{t_{1}} H\left(t, \widehat{x}(t), u(t), \lambda_{0}, p(t)\right) d t=\int_{t_{0}}^{t_{1}} H\left(t, \widehat{x}(t), \widehat{u}(t), \lambda_{0}, p(t)\right) d t .
$$

Это соотношение эквивалентно второму утверждению в следствии 4 на множестве, где функция $p(\cdot)$ непрерывна, а функция $\widehat{u}(\cdot)$ аппроксимативно непрерывна. Доказательство точно такое же, как в предыдущем следствии. Следствие 4 доказано.

2.5. Задача локальной управляемости. Пусть $\left[t_{0}, t_{1}\right]$ - отрезок прямой, $\mathscr{O}$ - открытое подмножество $\mathbb{R} \times \mathbb{R}^{n}, U$ - непустое подмножество $\mathbb{R}^{r}$, и пусть заданы отображение $\varphi: \mathscr{O} \times \operatorname{cl} U \rightarrow \mathbb{R}^{n}$, функции $g_{i}: \mathscr{O} \rightarrow \mathbb{R}, i=1, \ldots, m$, и $x_{0} \in \mathbb{R}^{n}$. Рассмотрим следующую управляемую систему с фазовыми ограничениями:

$$
\begin{gathered}
\dot{x}=\varphi(t, x, u), \quad x\left(t_{0}\right)=x_{0}, \quad u(t) \in U, \\
g_{i}(t, x(t)) \leqslant 0, \quad i=1, \ldots, m .
\end{gathered}
$$

Пару $(x(\cdot), u(\cdot)) \in A C\left(\left[t_{0}, t_{1}\right], \mathbb{R}^{n}\right) \times L_{\infty}\left(\left[t_{0}, t_{1}\right], \mathbb{R}^{r}\right)$ назовем допустимым процессом для управляемой системы $\left(\mathrm{P}_{5}\right)$, если $\Gamma(x(\cdot))=\left\{(t, x(t)) \mid t \in\left[t_{0}, t_{1}\right]\right\} \subset \mathscr{O}$, равенство $\dot{x}(t)=\varphi(t, x(t), u(t))$ и включение $u(t) \in U$ выполняются для почти всех $t \in\left[t_{0}, t_{1}\right]$, неравенства $g_{i}(t, x(t)) \leqslant 0, i=1, \ldots, m$, выполняются для всех $t \in\left[t_{0}, t_{1}\right]$ и $x\left(t_{0}\right)=x_{0}$.

Пусть $(\widehat{x}(\cdot), \widehat{u}(\cdot))$ - допустимый процесс в системе $\left(\mathrm{P}_{5}\right)$. Скажем, что система $\left(\mathrm{P}_{5}\right)$ локалъно управляема относителъно процесса $(\widehat{x}(\cdot), \widehat{u}(\cdot))$, если найдутся такие окрестность $W$ точки $\widehat{x}\left(t_{1}\right)$ и константа $K>0$, что для любой точки $\bar{x} \in W$ существует допустимый процесс $(\bar{x}(\cdot), \bar{u}(\cdot))$, для которого $\bar{x}\left(t_{1}\right)=\bar{x}$ и $\|\bar{x}(\cdot)-\widehat{x}(\cdot)\|_{C\left(\left[t_{0}, t_{1}\right], \mathbb{R}^{n}\right)} \leqslant K\left|\bar{x}-x_{1}\right|$.

Функцию

$$
H(t, x, u, p(\cdot))=(p(t), \varphi(t, x, u)),
$$

где $p(\cdot):\left[t_{0}, t_{1}\right] \rightarrow\left(\mathbb{R}^{n}\right)^{*}$, назовем функиией Понтрягина управляемой системы $\left(\mathrm{P}_{5}\right)$. 
СлЕдствиЕ 5 (достаточные условия локальной управляемости системы $\left(\mathrm{P}_{5}\right)$ ). Пусть $(\widehat{x}(\cdot), \widehat{u}(\cdot))$ - допустимый проиесс в системе $\left(\mathrm{P}_{5}\right)$. Тогда, если отображение $\varphi$ и функции $g_{i}, i=1, \ldots, m$, непрерывны вместе со своими частными производными по $x$ соответственно на $\mathscr{O} \times \operatorname{cl} U$ u $\mathscr{O}$ и если не существует не равных одновременно нулю функиии $p(\cdot):\left[t_{0}, t_{1}\right] \rightarrow\left(\mathbb{R}^{n}\right)^{*}$ ограниченной вариации, непрерьвной справа на $\left(t_{0}, t_{1}\right)$, и неотрицательной борелевской векторной меры $d \mu=\left(d \mu_{1}, \ldots, d \mu_{n}\right)$ на $\left[t_{0}, t_{1}\right]$, где $d \mu_{i}$ сосредоточена на множестве $T_{i}=\left\{t \in\left[t_{0}, t_{1}\right] \mid g_{i}(t, \widehat{x}(t))=0\right\}, i=1, \ldots, m$, удовлетворяющих соотношению

$$
p(t)=p\left(t_{1}\right)+\int_{t}^{t_{1}} H_{x}(\tau, \widehat{x}(\tau), \widehat{u}(\tau), p(\tau)) d \tau-\sum_{i=1}^{m} \int_{t}^{t_{1}} g_{i x}(\tau, \widehat{x}(\tau)) d \mu_{i}
$$

для всех $t \in\left[t_{0}, t_{1}\right]$ и соотношению

$$
\max _{u \in U} H(t, \widehat{x}(t), u, p(t))=H(t, \widehat{x}(t), \widehat{u}(t), p(t)),
$$

для почти всех $t \in\left[t_{0}, t_{1}\right]$, то система $\left(\mathrm{P}_{5}\right)$ локально управляема относительно процесса $(\widehat{x}(\cdot), \widehat{u}(\cdot))$.

ДокАЗАТЕЛЬство. Пусть $(\widehat{x}(\cdot), \widehat{u}(\cdot))$ - допустимый процесс в системе $\left(\mathrm{P}_{5}\right)$. Положим $X=C\left(\left[t_{0}, t_{1}\right], \mathbb{R}^{n}\right), Z_{1}=C\left(\left[t_{0}, t_{1}\right], \mathbb{R}^{n}\right) \times \mathbb{R}^{n} \times \mathbb{R}^{n}, Z_{2}=C\left(\left[t_{0}, t_{1}\right], \mathbb{R}^{m}\right)$, $V=\left\{x(\cdot) \in C\left(\left[t_{0}, t_{1}\right], \mathbb{R}^{n}\right) \mid \Gamma(x(\cdot)) \subset \mathscr{O}\right\}, \mathscr{U}=\left\{u(\cdot) \in L_{\infty}\left(\left[t_{0}, t_{1}\right], \mathbb{R}^{r}\right) \mid u(t) \in\right.$ $U$ для почти всех $\left.t \in\left[t_{0}, t_{1}\right]\right\}$ ( $\mathscr{U}$ рассматривается в топологии $L_{1}\left(\left[t_{0}, t_{1}\right], \mathbb{R}^{r}\right)$ ), $Q=\left\{y(\cdot) \in Z_{2} \mid y(t) \leqslant 0 \forall t \in\left[t_{0}, t_{1}\right]\right\}$, и пусть отображения $\Phi: V \times \mathscr{U} \rightarrow Z_{1}$ и $G: V \rightarrow Z_{2}$ определены соответственно формулами

$$
\Phi(x(\cdot), u(\cdot))(t)=\left(x(t)-x\left(t_{0}\right)-\int_{t_{0}}^{t} \varphi(\tau, x(\tau), u(\tau)) d \tau, x\left(t_{0}\right)-x_{0}, x\left(t_{1}\right)-x_{1}\right)
$$

для всех $t \in\left[t_{0}, t_{1}\right]$, где $x_{1}=\widehat{x}\left(t_{1}\right)$, и

$$
G(x(\cdot))(\cdot)=\left(g_{1}(\cdot, x(\cdot)), \ldots, g_{m}(\cdot, x(\cdot))\right) .
$$

Точно так же, как и при доказательстве следствия 4, проверяется, что для данных отображений выполнены условия 1)-4) теоремы о разрешимости и $\operatorname{codim} \operatorname{Im} \Phi_{x}(\widehat{x}(\cdot), \widehat{u}(\cdot))<\infty$. Условия следствия (о несуществовании функции $p(\cdot)$ и меры $d \mu_{i}$, удовлетворяющих приведенным соотношениям) есть расшифровка утверждений предложения 1, которая проводится совершенно так же, как и при доказательстве следствия 4. Но эти утверждения, в силу того же предложения 1, равносильны условию регулярности 5) в теореме о разрешимости, и, значит, все ее предположения относительно отображений $\Phi$ и $G$ выполнены. Согласно этой теореме и структуре отображения $\Phi$ существуют такие окрестность $V_{0}$ нуля в $\mathbb{R}^{n}$ и константа $K>0$, что для каждой точки $\bar{x} \in V_{0}$ найдется пара $(\bar{x}(\cdot), \bar{u}(\cdot)) \in C\left(\left[t_{0}, t_{1}\right], \mathbb{R}^{n}\right) \times \mathscr{U}$, для которой

$$
\begin{gathered}
\bar{x}(t)-\bar{x}\left(t_{0}\right)-\int_{t_{0}}^{t} \varphi(\tau, \bar{x}(\tau), \bar{u}(\tau)) d \tau=0, \\
\bar{x}\left(t_{0}\right)-x_{0}=0, \quad \bar{x}\left(t_{1}\right)-x_{1}=\bar{x},
\end{gathered}
$$

$G(\bar{x}(\cdot)) \in Q$ и $\|\bar{x}(\cdot)-\widehat{x}(\cdot)\|_{C\left(\left[t_{0}, t_{1}\right], \mathbb{R}^{n}\right)} \leqslant K\left|\bar{x}-x_{1}\right|$. 
Ясно, что $(\bar{x}(\cdot), \bar{u}(\cdot))$ - допустимая пара в системе $\left(\mathrm{P}_{5}\right)$ и в качестве окрестности $\widehat{x}\left(t_{1}\right)=x_{1}$ в определении локальной управляемости можно взять окрестность $W=x_{1}+V_{0}$. Следствие 5 доказано.

\section{3. Доказательство основной теоремы}

Сначала докажем одно вспомогательное предложение.

ПреДЛОЖЕНИЕ 2. Пусть $X, Z_{1}$ u $Z_{2}$ - банаховы пространства, $A_{i}: X \rightarrow$ $Z_{i}, i=1,2$, - линейные непрерывные операторь, $K_{i} \subset Z_{i}, i=1,2$, - непустые выпуклые замкнутые множества, $0 \in \operatorname{int}\left(\operatorname{Im} A_{1}-K_{1}\right) u \operatorname{int} K_{2} \neq \varnothing$. Тогда $\operatorname{int}\left(\operatorname{Im}\left(A_{1}, A_{2}\right)-\left(K_{1}, K_{2}\right)\right) \neq \varnothing$, где оператор $\left(A_{1}, A_{2}\right): X \rightarrow Z_{1} \times Z_{2}$ (норма в $Z_{1} \times Z_{2}$ понимается как сумма норм) действует по правилу $\left(A_{1}, A_{2}\right) x=$ $\left(A_{1} x, A_{2} x\right) u\left(K_{1}, K_{2}\right)=\left\{\left(z_{1}, z_{2}\right) \in Z_{1} \times Z_{2} \mid z_{i} \in K_{i}, i=1,2\right\}$.

Доказательство. Пусть $\bar{z}_{2} \in \operatorname{int} K_{2}$. Тогда существует такое $\varepsilon>0$, что $B_{Z_{2}}\left(\bar{z}_{2}, \varepsilon\right) \subset K_{2}$. Рассмотрим многозначное отображение $\Psi_{1}$ из $X$ в $Z_{1}$, определенное формулой $\Psi_{1} x=A_{1} x-K_{1}$. Так как по условию $0 \in \operatorname{int} \operatorname{Im} \Psi_{1}$, то по теореме Робинсона-Урсеску (см. [14; гл. 3]) найдутся такие $a>0, \delta>0$, что для всех $z_{1} \in B_{Z_{1}}(0, \delta)$ справедливо неравенство $d\left(0, \Psi_{1}^{-1}\left(z_{1}\right)\right) \leqslant \operatorname{ad}\left(z_{1}, \Psi_{1}(0)\right)$. Поскольку $0 \in \Psi_{1}(0)$, то, продолжая это неравенство, будем иметь

$$
d\left(0, \Psi_{1}^{-1}\left(z_{1}\right)\right) \leqslant a\left\|z_{1}\right\|_{Z_{1}} \leqslant a \delta
$$

где считаем, что $\delta \leqslant \min \left(\varepsilon /\left(4 a\left\|A_{2}\right\|\right), \varepsilon / 2\right)$. Из (3.1) следует, что для любого $z_{1} \in B_{Z_{1}}(0, \delta)$ найдется элемент $x\left(z_{1}\right) \in \Psi_{1}^{-1}\left(z_{1}\right)$ такой, что $\left\|x\left(z_{1}\right)\right\|_{X} \leqslant 2 a \delta \leqslant$ $\varepsilon /\left(2\left\|A_{2}\right\|\right)$ и, значит, $\left\|A_{2} x\left(z_{1}\right)\right\|_{Z_{2}} \leqslant \varepsilon / 2$.

Покажем, что $\bar{z}=\left(0, \bar{z}_{2}\right) \in \operatorname{int}\left(\operatorname{Im}\left(A_{1}, A_{2}\right)-\left(K_{1}, K_{2}\right)\right)$. Действительно, пусть $z=\left(z_{1}, z_{2}\right) \in B_{Z_{1} \times Z_{2}}(\bar{z}, \delta)$. Тогда $z_{1} \in B_{Z_{1}}(0, \delta)$ и по доказанному $z_{1} \in$ $\Psi_{1}\left(x\left(z_{1}\right)\right)=A_{1} x\left(z_{1}\right)-K_{1}$. Далее, $\left\|A_{2} x\left(z_{1}\right)-z_{2}-\bar{z}_{2}\right\|_{Z_{2}} \leqslant\left\|A_{2} x\left(z_{1}\right)\right\|_{Z_{2}}+\| z_{2}-$ $\bar{z}_{2} \|_{Z_{2}} \leqslant \varepsilon / 2+\varepsilon / 2=\varepsilon$, т. е. $A_{2} x\left(z_{1}\right)-z_{2} \in B_{Z_{2}}\left(\bar{z}_{2}, \varepsilon\right) \subset K_{2}$, или $z_{2} \in A_{2} x\left(z_{1}\right)-K_{2}$. Таким образом, $B_{Z_{1} \times Z_{2}}(\bar{z}, \delta) \subset \operatorname{Im}\left(A_{1}, A_{2}\right)-\left(K_{1}, K_{2}\right)$ и, следовательно, внутренность множества $\operatorname{Im}\left(A_{1}, A_{2}\right)-\left(K_{1}, K_{2}\right)$ непуста. Предложение доказано.

ДокАЗАТЕЛЬСТво осНОвНоЙ ТЕОРЕмЫ. Поскольку отображение $\left(f_{0}, F\right)$ гладко-аппроксимативно-выпукло в точке $(\widehat{x}, \widehat{u})$, то, очевидно, что таково и отображение $\Phi=\left(f_{0}-f_{0}(\widehat{x}, \widehat{u}), F\right)$. Так как $\operatorname{codim} \operatorname{Im} F_{x}(\widehat{x}, \widehat{u})<\infty$, то легко видеть, что и $\operatorname{codim} \operatorname{Im} \Phi_{x}(\widehat{x}, \widehat{u})<\infty$. Покажем, что если $0 \in \operatorname{int}\left(\operatorname{Im}\left(\Phi_{x}(\widehat{x}, \widehat{u})\right.\right.$, $\left.\left.G_{x}(\widehat{x})\right)+(\operatorname{co} \Phi(\widehat{x}, \mathscr{U}), G(\widehat{x})-Q)\right)$, то это приводит к противоречию с тем, что $(\widehat{x}, \widehat{u})$ - локальный минимум в задаче $(\mathrm{P})$.

Действительно, в этих предположениях выполнены условия теоремы о разрешимости $\left(\right.$ с $\left.Z_{1}=\mathbb{R} \times Y_{1}, Z_{2}=Y_{2}\right)$, согласно которой для всех достаточно малых $\varepsilon>0$ найдутся такие $x_{\varepsilon}$ и $u_{\varepsilon}$, что $\Phi\left(x_{\varepsilon}, u_{\varepsilon}\right)=(-\varepsilon, 0)$ и $G\left(x_{\varepsilon}\right) \in Q$, или $\left.f_{0}\left(x_{\varepsilon}, u_{\varepsilon}\right)\right)=f_{0}(\widehat{x}, \widehat{u})-\varepsilon, F\left(x_{\varepsilon}, u_{\varepsilon}\right)=0$ и $G\left(x_{\varepsilon}\right) \in Q$. При этом $x_{\varepsilon} \rightarrow \widehat{x}$ при $\varepsilon \rightarrow 0$ (в силу оценки в теореме о разрешимости). Другими словами, в любой окрестности точки $\widehat{x}$ существует элемент $x_{\varepsilon}$ такой, что пара $\left(x_{\varepsilon}, u_{\varepsilon}\right)$ допустима в задаче $(\mathrm{P})$, а значение $f_{0}$ на этой паре меньше, чем на $(\widehat{x}, \widehat{u})$, что невозможно.

Итак, $0 \notin \operatorname{int}\left(\operatorname{Im}\left(\Phi_{x}(\widehat{x}, \widehat{u}), G^{\prime}(\widehat{x})\right)+(\operatorname{co} \Phi(\widehat{x}, \mathscr{U}), G(\widehat{x})-Q)\right)$. Рассмотрим два случая. 
1) $0 \notin \operatorname{int}\left(\operatorname{Im} \Phi_{x}(\widehat{x}, \widehat{u})+\operatorname{co} \Phi(\widehat{x}, \mathscr{U})\right)$. Пространство $\operatorname{Im} \Phi_{x}(\widehat{x}, \widehat{u})$ замкнуто, как подпространство конечной коразмерности, являющееся образом линейного непрерывного оператора. Рассмотрим факторпространство $\left(\mathbb{R} \times Y_{1}\right) / \operatorname{Im} \Phi_{x}(\widehat{x}, \widehat{u})$, и пусть $\pi: \mathbb{R} \times Y_{1} \rightarrow\left(\mathbb{R} \times Y_{1}\right) / \operatorname{Im} \Phi_{x}(\widehat{x}, \widehat{u})$ - каноническая проекция. Тогда $0 \notin$ $\operatorname{int} \pi \operatorname{co} \Phi(\widehat{x}, \mathscr{U})$ в силу непрерывности отображения $\pi$. Если int $\pi \operatorname{co} \Phi(\widehat{x}, \mathscr{U}) \neq \varnothing$, то, применяя (конечномерную) теорему отделимости к выпуклым множествам $\{0\}$ и int $\pi \operatorname{co} \Phi(\widehat{x}, \mathscr{U})$, найдем такой ненулевой элемент $w^{*} \in\left(\left(\mathbb{R} \times Y_{1}\right) / \operatorname{Im} \Phi_{x}(\widehat{x}, \widehat{u})\right)^{*}$, что $\left\langle w^{*}, \pi \Phi(\widehat{x}, u)\right\rangle \geqslant 0$ для всех $u \in \mathscr{U}$, и ясно, что $\left\langle w^{*}, \pi \Phi_{x}(\widehat{x}, \widehat{u}) x\right\rangle=0$ для любого $x \in X$. Если обозначить $\left(\lambda_{0}, \lambda_{1}\right)=\pi^{*} w^{*} \in(\mathbb{R} \times Y)^{*}\left(\left(\lambda_{0}, \lambda_{1}\right) \neq 0\right.$, так как $\pi$ сюръективно), то последние соотношения перепишутся так: $\lambda_{0} f_{0}(\widehat{x}, u)+$ $\langle\lambda, F(\widehat{x}, u)\rangle \geqslant \lambda_{0} f_{0}(\widehat{x}, \widehat{u})$ для всех $u \in \mathscr{U}$ и $\lambda_{0}\left\langle f_{0}^{\prime}(\widehat{x}, \widehat{u}), x\right\rangle+\left\langle\lambda_{1}, F_{x}(\widehat{x}, \widehat{u}) x\right\rangle=0$ для всех $x \in X$, что есть утверждение теоремы с $\bar{\lambda}=\left(\lambda_{0}, \lambda_{1}, 0\right)$.

Если же int $\pi \operatorname{co} \Phi(\widehat{x}, \mathscr{U})=\varnothing$, то выпуклое множество $\pi$ со $\Phi(\widehat{x}, \mathscr{U})$ содержится в некоторой гиперплоскости, т. е. существует такой ненулевой элемент $w^{*} \in\left((\mathbb{R} \times Y) / \operatorname{Im} \Phi_{x}(\widehat{x}, \widehat{u})\right)^{*}$, что $\left\langle w^{*}, \pi \Phi(\widehat{x}, u)\right\rangle=0$ для всех $u \in \mathscr{U}$, и ясно, что $\left\langle w^{*}, \pi \Phi_{x}(\widehat{x}, \widehat{u}) x\right\rangle=0$ для любого $x \in X$. Снова обозначая $\left(\lambda_{0}, \lambda_{1}\right)=\pi^{*} w^{*}$, приходим, аналогично предыдущему, к утверждению теоремы с $\bar{\lambda}=\left(\lambda_{0}, \lambda_{1}, 0\right)$.

2) $0 \in \operatorname{int}\left(\operatorname{Im} \Phi_{x}(\widehat{x}, \widehat{u})+\operatorname{co} \Phi(\widehat{x}, \mathscr{U})\right)$. В этом случае внутренность множества $\operatorname{Im}\left(\Phi_{x}(\widehat{x}, \widehat{u}), G^{\prime}(\widehat{x})\right)+(\operatorname{co} \Phi(\widehat{x}, \mathscr{U}), G(\widehat{x})-Q)$ непуста, что сразу следует из предложения 2 , если обозначить $A_{1}=\Phi_{x}(\widehat{x}, \widehat{u}), A_{2}=G^{\prime}(\widehat{x}), K_{1}=-\operatorname{co} \Phi(\widehat{x}, \mathscr{U})$ и $K_{2}=-G(\widehat{x})+Q$. По условию, нуль этой внутренности не принадлежит, и, значит, по теореме отделимости найдется ненулевой функционал $\mu \in\left(\mathbb{R} \times Y_{1} \times Y_{2}\right)^{*}$ такой, что $\langle\mu, y\rangle \geqslant 0$ для любого $y \in \operatorname{Im}\left(\Phi_{x}(\widehat{x}, \widehat{u}), G^{\prime}(\widehat{x})\right)+(\operatorname{co} \Phi(\widehat{x}, \mathscr{U}), G(\widehat{x})-Q)$. Следовательно, существует ненулевой набор $\bar{\lambda}=\left(\lambda_{0}, \lambda_{1}, \lambda_{2}\right) \in \mathbb{R} \times Y_{1}^{*} \times Y_{2}^{*}$ такой, что для всех $x \in X$, всех $\left(\alpha, y_{1}\right) \in \operatorname{co}\left(f_{0}(\widehat{x}, \mathscr{U})-f_{0}(\widehat{x}, \widehat{u}), F(\widehat{x}, \mathscr{U})\right)$ и всех $y_{2} \in Q$ справедливо неравенство

$$
\lambda_{0}\left(\left\langle f_{0 x}(\widehat{x}, \widehat{u}), x\right\rangle+\alpha\right)+\left\langle\lambda_{1}, F_{x}(\widehat{x}, \widehat{u}) x+y_{1}\right\rangle+\left\langle\lambda_{2}, G^{\prime}(\widehat{x}) x+G(\widehat{x})-y_{2}\right\rangle \geqslant 0 .
$$

Полагая здесь $\alpha=0, y_{1}=0$ и $y_{2}=G(\widehat{x})$, получаем, что линейный функционал $\lambda_{0} f_{0 x}(\widehat{x}, \widehat{u})+\left(F_{x}(\widehat{x}, \widehat{u})\right)^{*} \lambda_{1}+\left(G^{\prime}(\widehat{x})\right)^{*} \lambda_{2}$ неотрицателен на $X$ и, значит, он нулевой. Равенство (I) доказано.

Пусть в $(3.2) x=0$ и $y_{2}=G(\widehat{x})$. Для каждого $u \in \mathscr{U}$ полагая $\alpha=f_{0}(\widehat{x}, u)-$ $f_{0}(\widehat{x}, \widehat{u})$ и $y_{1}=F(\widehat{x}, u)$, получаем соотношение $(\mathrm{II})$. Если положить здесь $y_{1}=0$, то, поскольку $f_{0}(\widehat{x}, u)-f_{0}(\widehat{x}, \widehat{u}) \geqslant 0$, получаем, что $\lambda_{0} \geqslant 0$.

Наконец, если в (3.2) $x=0, \alpha=0, y_{1}=0$, то приходим к тому, что $\lambda_{2} \in$ $N_{Q}(G(\widehat{x}))$. Теорема доказана.

ДоКАЗАТЕЛЬСТвО ПРЕДЛОЖЕНИЯ 1. Пусть выполнены утверждения предложения 1. Покажем, что справедливы условия регулярности в теореме о разрешимости. Действительно, если эти условия не выполняются, то, практически дословно повторяя доказательство основной теоремы, приходим к тому, что существуют линейные функционалы $\lambda_{1} \in Z_{1}^{*}$ и $\lambda_{2} \in N_{Q}(G(\widehat{x}))$, не равные одновременно нулю, такие, что справедливы соотношения $(*)$ и $(* *)-$ в противоречие с предположением.

Обратно, пусть выполнены условия регулярности в теореме о разрешимости. Предположим, что не выполняются утверждения предложения 1, т. е. существуют не равные одновременно нулю линейные функционалы $\lambda_{1} \in Z_{1}^{*}$ и 
$\lambda_{2} \in N_{Q}(G(\widehat{x}))$ такие, что справедливы соотношения $(*)$ и $(* *)$. Тогда из $(* *)$ и того, что $\lambda_{2} \in N_{Q}(G(\widehat{x}))$, следует, что для любых $z_{1} \in \operatorname{co} \Phi(\widehat{x}, \mathscr{U})$ и $z_{2} \in Q$ выполнено неравенство $\left\langle\lambda_{1}, z_{1}\right\rangle+\left\langle\lambda_{2}, G(\widehat{x})-z_{2}\right\rangle \geqslant 0$. Отсюда и из $(*)$ получаем, что

$$
\left\langle\lambda_{1}, \Phi_{x}(\widehat{x}, \widehat{u}) x+z_{1}\right\rangle+\left\langle\lambda_{2}, G^{\prime}(\widehat{x}) x+G(\widehat{x})-z_{2}\right\rangle \geqslant 0
$$

для всех $x \in X, z_{1} \in \operatorname{co} \Phi(\widehat{x}, \mathscr{U})$ и $z_{2} \in Q$. Иначе говоря, если обозначить $\mu=\left(\lambda_{1}, \lambda_{2}\right)$, то для всех $y \in M=\operatorname{Im}\left(\Phi_{x}(\widehat{x}, \widehat{u}), G^{\prime}(\widehat{x})\right)+(\operatorname{co} \Phi(\widehat{x}, \mathscr{U}), G(\widehat{x})-Q)$ справедливо неравенство $\langle\mu, y\rangle \geqslant 0$. Но $0 \in \operatorname{int} M$ и, значит, $\mu$ - нулевой функционал в противоречие с предположением. Предложение 1 доказано.

ДОКАЗАТЕЛЬСТВО ТЕОРЕМЫ О РАЗРЕШИМОСТИ. В основе доказательства лежат модифицированный метод Ньютона и теорема Брауэра о неподвижной точке.

1. Предварителъные построения. Начнем с формулировки вспомогательного утверждения, которое непосредственно вытекает из следствия 2.101 в [15] и в наших терминах звучит так: существует элемент $h \in X$ такой, что $\Phi_{x}(\widehat{x}, \widehat{u}) h \in$ $-\operatorname{co} \Phi(\widehat{x}, \mathscr{U})$, т. е.

$$
\Phi_{x}(\widehat{x}, \widehat{u}) h=-\sum_{i=1}^{m} \beta_{i} \Phi\left(\widehat{x}, v_{i}\right)
$$

для некоторых $v_{i} \in \mathscr{U}, \beta_{i} \geqslant 0, i=1, \ldots, m, \sum_{i=1}^{m} \beta_{i}=1$, и

$$
G^{\prime}(\widehat{x}) h \in \operatorname{int}(Q-G(\widehat{x})) .
$$

Положим $\Lambda_{1}=\Phi_{x}(\widehat{x}, \widehat{u})$. Подпространство $\operatorname{Im} \Lambda_{1}$ замкнуто, как подпространство конечной коразмерности, являющееся образом линейного непрерывного оператора. Следовательно, $Z_{1}$ может быть представлено как прямая сумма замкнутого подпространства $\operatorname{Im} \Lambda_{1}$ и некоторого конечномерного подпространства $L$. Тогда существуют непрерывные проекторы $P_{1}: Z_{1} \rightarrow \operatorname{Im} \Lambda_{1}$ и $P_{2}: Z_{1} \rightarrow L$. В силу условий регулярности, $0 \in \operatorname{int}\left(\operatorname{Im} \Lambda_{1}+\operatorname{co} \Phi(\widehat{x}, \mathscr{U})\right)$, и поэтому $0 \in \operatorname{int} P_{2}\left(\operatorname{Im} \Lambda_{1}+\operatorname{co} \Phi(\widehat{x}, \mathscr{U})\right)=\operatorname{int} P_{2}(\operatorname{co} \Phi(\widehat{x}, \mathscr{U}))$. Поскольку $L=\operatorname{Im} P_{2}$ конечномерно, то найдется конечный набор векторов из $P_{2}(\operatorname{co} \Phi(\widehat{x}, \mathscr{U}))$, выпуклая коническая оболочка которых совпадает с $L$. Но тогда существуют такие $u_{i} \in \mathscr{U}, i=1, \ldots, k$, что выпуклая коническая оболочка векторов $w_{i}=$ $P_{2} \Phi\left(\widehat{x}, u_{i}\right), i=1, \ldots, k$, также совпадает с $L$. Можно считать, что $k \geqslant m$ и $u_{i}=v_{i}, i=1, \ldots, m$.

Обозначим $\bar{u}=\left(\widehat{u}, u_{1}, \ldots, u_{k}\right)$. Если $\left(\alpha_{0}, \alpha_{1}, \ldots, \alpha_{k}\right) \in \Sigma^{k+1}$, то $\alpha_{0}=1-\sum_{i=1}^{k} \alpha_{i}$, и поэтому далее будем иметь дело с векторами $\alpha=\left(\alpha_{1}, \ldots, \alpha_{k}\right)$, которые пробегают симплекс $\Sigma_{0}^{k}=\left\{\alpha=\left(\alpha_{1}, \ldots, \alpha_{k}\right) \in \mathbb{R}_{+}^{k} \mid \sum_{i=1}^{k} \alpha_{i} \leqslant 1\right\}$. Микс, построенный по $\bar{u},\left(\alpha_{0}, \alpha_{1}, \ldots, \alpha_{k}\right) \in \Sigma^{k+1}$ и $\delta>0$, по-прежнему обозначаем $M(\alpha, \delta, \bar{u})$, но уже $\alpha=\left(\alpha_{1}, \ldots, \alpha_{k}\right) \in \Sigma_{0}^{k}$ (в частности, $\left.M(0, \delta, \bar{u})=\widehat{u}\right)$.

Схема дальнейших рассуждений такова. Сначала, используя модифицированный метод Ньютона, строим семейство решений уравнения $P_{1} \Phi(x, M(\alpha, \delta, \bar{u}))=$ $P_{1} z$. Точнее говоря, для каждых $\delta>0, \xi$, близких к $\widehat{x}$, и малых по норме 
$z \in Z_{1}$ и $\alpha \in \Sigma_{0}^{k}$ строится отображение $(\xi, \alpha, z, \delta) \mapsto \varphi(\xi, \alpha, z, \delta)$, непрерывное по $(\xi, \alpha, z)$, такое, что

$$
P_{1} \Phi(\varphi(\xi, \alpha, z, \delta), M(\alpha, \delta, \bar{u}))=P_{1} z
$$

и доказывается оценка

$$
\|\varphi(\xi, \alpha, z, \delta)-\xi\|_{X} \leqslant K_{1}\left\|P_{1}(z-\Phi(\xi, M(\alpha, \delta, \bar{u})))\right\|_{Z_{1}}
$$

с некоторым $K_{1}>0$.

Далее по $z$ выбираем $\delta(z), \xi(z)$, а затем, используя теорему Брауэра о неподвижной точке, находим $\alpha(z)$ такое, что выполняются равенство

$$
P_{2} \Phi(\varphi(\xi(z), \alpha(z), z, \delta(z)), M(\alpha(z), \delta(z), \bar{u}))=P_{2} z
$$

и включение $\varphi(\xi(z), \alpha(z), z, \delta(z)) \in Q$.

Обозначая $x(z)=\varphi(\xi(z), \alpha(z), z, \delta(z))$ и $u(z)=M(\alpha(z), \delta(z), \bar{u})$, приходим, согласно (3.5) и (3.7), к соотношениям $P_{1} \Phi(x(z), u(z))=P_{1} z$ и $P_{2} \Phi(x(z), u(z))=$ $P_{2} z$, которые равносильны нужному уравнению: $\Phi(x(z), u(z))=z$. Соответствующая оценка в теореме устанавливается по ходу дела.

2. Построение семейства решений уравнения $P_{1} \Phi(x, M(\alpha, \delta, \bar{u}))=P_{1} z$. Заметим сначала, что из теоремы Майкла о непрерывном селекторе и теоремы Банаха об открытом отображении следует существование непрерывного правого обратного к отображению $P_{1} \Lambda_{1}: X \rightarrow \operatorname{Im} \Lambda_{1}$, а именно, такого непрерывного отображения $R_{1}: \operatorname{Im} \Lambda_{1} \rightarrow X$ и константы $\gamma_{1}>0$, что $P_{1} \Lambda_{1} R_{1}(z)=z$ и $\left\|R_{1}(z)\right\|_{X} \leqslant \gamma_{1}\|z\|_{Z_{1}}$ для всех $z \in \operatorname{Im} \Lambda_{1}$.

Пусть $\varepsilon_{1}>0$ таково, что $\varepsilon_{1} \gamma_{1}\left\|P_{1}\right\|<1$. Из непрерывности микса и гладкости $\Phi$ следует существование таких $\delta_{1}=\delta_{1}\left(\varepsilon_{1}\right)>0$ и $\delta_{2}=\delta_{2}\left(\varepsilon_{1}\right)>0$, что для всех $x, x^{\prime} \in U_{X}\left(\widehat{x}, \delta_{1}\right), \alpha \in U_{\mathbb{R}^{k}}\left(0, \delta_{2}\right) \cap \Sigma_{0}^{k}$ (уменьшая $\delta_{2}$, считаем, что $U_{\mathbb{R}^{k}}\left(0, \delta_{2}\right) \cap$ $\left.\Sigma_{0}^{k}=U_{\mathbb{R}^{k}}\left(0, \delta_{2}\right) \cap \mathbb{R}_{+}^{k}\right)$ и $\delta>0$ справедливо неравенство

$$
\begin{gathered}
\left\|\Phi(x, M(\alpha, \delta, \bar{u}))-\Phi\left(x^{\prime}, M(\alpha, \delta, \bar{u})\right)-\Lambda_{1}\left(x-x^{\prime}\right)\right\|_{Z_{1}} \\
\leqslant \varepsilon_{1}\left\|x-x^{\prime}\right\|_{X}=\frac{\theta}{\gamma_{1}\left\|P_{1}\right\|}\left\|x-x^{\prime}\right\|_{X}
\end{gathered}
$$

где $0<\theta=\varepsilon_{1} \gamma_{1}\left\|P_{1}\right\|<1$. Обозначим $\delta_{3}=\left(1-\theta_{1}\right) \delta_{1} /\left(4 \gamma_{1}\left\|P_{1}\right\|\right)$. Вследствие непрерывности микса и $\Phi$ отображение $(x, \alpha) \mapsto \Phi(x, M(\alpha, \delta, \bar{u}))$ непрерывно в окрестности $(\widehat{x}, 0)$ (пересеченной с $X \times \mathbb{R}_{+}^{k}$ ) равномерно по $\delta$, и так как $\Phi(\widehat{x}, M(0, \delta, \bar{u}))=0$, то найдется $0<\delta_{4}<\delta_{1} / 4$ такое, что (при уменьшении в случае необходимости $\delta_{2}$ ) будут выполняться (равномерно по $\delta$ ) соотношения $\|\Phi(\xi, M(\alpha, \delta, \bar{u}))-\Phi(\widehat{x}, M(\alpha, \delta, \bar{u}))\|_{Z_{1}}<\delta_{3}$, если $(\xi, \alpha) \in U_{X}\left(\widehat{x}, \delta_{4}\right) \times\left(U_{\mathbb{R}^{k}}\left(0, \delta_{2}\right) \cap\right.$ $\left.\mathbb{R}_{+}^{k}\right)$, и $\|\Phi(\widehat{x}, M(\alpha, \delta, \bar{u}))\|_{Z_{1}}<\delta_{3}$, если $\alpha \in U_{\mathbb{R}^{k}}\left(0, \delta_{2}\right) \cap \mathbb{R}_{+}^{k}$.

Пусть $\xi \in U_{X}\left(\widehat{x}, \delta_{4}\right), \alpha \in U_{\mathbb{R}^{k}}\left(0, \delta_{2}\right) \cap \mathbb{R}_{+}^{k}, z \in U_{Z_{1}}\left(0, \delta_{3}\right)$ и $\delta>0$. Рассмотрим последовательность (модифицированный метод Ньютона)

$$
x_{n}=x_{n-1}+R_{1} P_{1}\left(z-\Phi\left(x_{n-1}, M(\alpha, \delta, \bar{u})\right)\right), \quad n \in \mathbb{N}, \quad x_{0}=\xi .
$$

Покажем, что эта последовательность содержится в шаре $U_{X}\left(\widehat{x}, \delta_{1}\right)$ и фундаментальна. Первое доказываем по индукции. Ясно, что $x_{0}=\xi \in U_{X}\left(\widehat{x}, \delta_{1}\right)$. 
Пусть $x_{i} \in U_{X}\left(\widehat{x}, \delta_{1}\right), 1 \leqslant i \leqslant n$. Покажем, что $x_{n+1} \in U_{X}\left(\widehat{x}, \delta_{1}\right)$. Используя последовательно (3.9) (с оценкой для $R_{1}$ ), равенство

$$
P_{1} \Lambda_{1}\left(x_{n}-x_{n-1}\right)-P_{1} z+P_{1} \Phi\left(x_{n-1}, M(\alpha, \delta, \bar{u})\right)=0,
$$

которое следует из (3.9) после применения к обеим частям оператора $P_{1} \Lambda_{1}$, неравенство (3.8) и затем итерируя процедуру, будем иметь

$$
\begin{aligned}
& \left\|x_{n+1}-x_{n}\right\|_{X} \leqslant \gamma_{1}\left\|P_{1}\left(z-\Phi\left(x_{n}, M(\alpha, \delta, \bar{u})\right)\right)\right\|_{Z_{1}} \\
& \quad=\gamma_{1}\left\|P_{1} \Phi\left(x_{n}, M(\alpha, \delta, \bar{u})\right)-P_{1} \Phi\left(x_{n-1}, M(\alpha, \delta, \bar{u})\right)-P_{1} \Lambda_{1}\left(x_{n}-x_{n-1}\right)\right\|_{Z_{1}} \\
& \quad \leqslant \gamma_{1}\left\|P_{1}\right\|\left\|\Phi\left(x_{n}, M(\alpha, \delta, \bar{u})\right)-\Phi\left(x_{n-1}, M(\alpha, \delta, \bar{u})\right)-\Lambda_{1}\left(x_{n}-x_{n-1}\right)\right\|_{Z_{1}} \\
& \quad \leqslant \theta\left\|x_{n}-x_{n-1}\right\|_{X} \leqslant \cdots \leqslant \theta^{n}\left\|x_{1}-\xi\right\|_{X} .
\end{aligned}
$$

Теперь, используя неравенство треугольника, (3.11), формулу для суммы геометрической прогрессии, (3.2) (при $n=1$ и снова с оценкой для $R_{1}$ ), выбор $\xi$, $\alpha$ и $z$, получим

$$
\begin{aligned}
& \left\|x_{n+1}-\widehat{x}\right\|_{X} \leqslant\left\|x_{n+1}-x_{n}\right\|_{X}+\cdots+\left\|x_{1}-\widehat{x}\right\|_{X} \\
& \leqslant\left\|x_{n+1}-x_{n}\right\|_{X}+\cdots+\left\|x_{1}-\xi\right\|_{X}+\|\xi-\widehat{x}\|_{X} \\
& \leqslant\left(\theta^{n}+\theta^{n-1}+\cdots+1\right)\left\|x_{1}-\xi\right\|_{X}+\|\xi-\widehat{x}\|_{X} \\
& <\frac{\gamma_{1}}{1-\theta}\left\|P_{1}(z-\Phi(\xi, M(\alpha, \delta, \bar{u})))\right\|_{Z_{1}}+\|\xi-\widehat{x}\|_{X} \\
& \leqslant \frac{\gamma_{1}\left\|P_{1}\right\|}{1-\theta}\|z\|_{Z_{1}}+\frac{\gamma_{1}\left\|P_{1}\right\|}{1-\theta}\|\Phi(\xi, M(\alpha, \delta, \bar{u}))-\Phi(\widehat{x}, M(\alpha, \delta, \bar{u}))\|_{Z_{1}} \\
& \quad+\frac{\gamma_{1}\left\|P_{1}\right\|}{1-\theta}\|\Phi(\widehat{x}, M(\alpha, \delta, \bar{u}))\|_{Z_{1}}+\|\xi-\widehat{x}\|_{X} \\
& \quad<\frac{\delta_{1}}{4}+\frac{\delta_{1}}{4}+\frac{\delta_{1}}{4}+\frac{\delta_{1}}{4}=\delta_{1} .
\end{aligned}
$$

Таким образом, $x_{n+1} \in U_{X}\left(\widehat{x}, \delta_{1}\right)$, и, значит, вся последовательность $\left\{x_{n}\right\}$ принадлежит этому шару.

Используя те же соображения, что и при доказательстве неравенства (3.12), имеем для любых $m, n \in \mathbb{N}$

$$
\begin{aligned}
\left\|x_{n+m}-x_{n}\right\|_{X} & \leqslant\left\|x_{n+m}-x_{n+m-1}\right\|_{X} \leqslant \cdots \leqslant\left\|x_{n+1}-x_{n}\right\|_{X} \\
& \leqslant\left(\theta_{1}^{n+m-1}+\cdots+\theta_{1}^{n}\right)\left\|x_{1}-\xi\right\|_{X} \\
& \leqslant \frac{\theta^{n} \gamma_{1}}{1-\theta}\left\|P_{1}(z-\Phi(\xi, M(\alpha, \delta, \bar{u})))\right\|_{Z_{1}} \leqslant \frac{3 \theta^{n} \delta_{1}}{4},
\end{aligned}
$$

т. е. последовательность $\left\{x_{n}\right\}$ фундаментальна.

Положим $\varphi(\xi, \alpha, z, \delta)=\lim _{n \rightarrow \infty} x_{n}$. Из (3.12) сразу следует, что $\varphi(\xi, \alpha, z, \delta) \in$ $U_{X}\left(\widehat{x}, \delta_{1}\right)$. Переходя к пределу при $n \rightarrow \infty$ в равенстве (3.10), получаем соотношение (3.5). Полагая в (3.13) $n=0$ и переходя к пределу при $m \rightarrow \infty$, приходим к оценке $(3.6)$ с $K_{1}=\gamma_{1} /(1-\theta)$.

Отображение $(\xi, \alpha, z) \mapsto \varphi(\xi, \alpha, z, \delta)$ непрерывно на $U_{X}\left(\widehat{x}, \delta_{4}\right) \times\left(U_{\mathbb{R}^{k}}\left(0, \delta_{2}\right) \cap\right.$ $\left.\mathbb{R}_{+}^{k}\right) \times U_{Z_{1}}\left(0, \delta_{3}\right)$ для каждого $\delta>0$. Действительно, функции $x_{n}$, как функции $\xi, \alpha$ и $z$, непрерывны на этом множестве при каждом $\delta>0$ в силу непрерывности $R_{1}$, свойств микса и отображения $\Phi$. Переходя к пределу в $(3.13)$ 
при $m \rightarrow \infty$, получаем, что функции $x_{n}$ равномерно по $\xi, \alpha$ и $z$ на том же множестве сходятся к $\varphi$ и, значит, само отображение $\varphi$ непрерывно на $U_{X}\left(\widehat{x}, \delta_{4}\right) \times$ $\left(U_{\mathbb{R}^{k}}\left(0, \delta_{2}\right) \cap \mathbb{R}_{+}^{k}\right) \times U_{Z_{1}}\left(0, \delta_{3}\right)$.

ЗАмЕчАниЕ 2. Неравенство (3.8) является основным соотношением для применения метода Ньютона. Из него следует, что если для всех $\alpha \in \Sigma_{0}^{k}$ микс не выходит за пределы некоторого множества $\mathscr{U}_{0} \subset \mathscr{U}$, то достаточно, чтобы условия гладкости отображения $\Phi$ выполнялись только для этого множества.

3. Доказательство соотношения (3.7). Определим оператор $\Lambda_{2}: \mathbb{R}^{k} \rightarrow Z_{1}$ по формуле $\Lambda_{2} \alpha=\sum_{i=1}^{k} \alpha_{i} \Phi\left(\widehat{x}, u_{i}\right)$, где $\alpha=\left(\alpha_{1}, \ldots, \alpha_{k}\right)$. Тогда $P_{2} \Lambda_{2} \mathbb{R}_{+}^{k}=L$. Действительно, по доказанному выше, для любого $z \in L$ найдется вектор $\alpha(z)=$ $\left(\alpha_{1}(z), \ldots, \alpha_{k}(z)\right) \in \mathbb{R}_{+}^{k}$ такой, что $z=\sum_{i=1}^{k} \alpha_{i}(z) w_{i}=\sum_{i=1}^{k} \alpha_{i}(z) \Phi\left(\widehat{x}, u_{i}\right)=\Lambda_{2} \alpha(z)$ и, значит, $z=P_{2} z=P_{2} \Lambda_{2} \alpha(z)$.

По тем же соображениям, что и выше, существует непрерывный правый обратный к $P_{2} \Lambda_{2}$, т. е. такие непрерывное отображение $R_{2}: L \rightarrow \mathbb{R}_{+}^{k}$ ( $L$ рассматривается в индуцированной норме, в $\mathbb{R}^{k}$ евклидова норма $\left.|\cdot|\right)$ и константа $\gamma_{2}>0$, что $P_{2} \Lambda_{2} R_{2}(w)=w$ и $\left|R_{2}(w)\right| \leqslant \gamma_{2}\|w\|_{Z_{1}}$ для всех $w \in L$ (хотя в данной, конечномерной, ситуации существование правого обратного нетрудно доказать и непосредственно).

Теперь определим некоторые константы. Из (3.4) следует существование такого $\eta>0$, что

$$
G^{\prime}(\widehat{x}) h+B_{Z_{2}}(0, \eta) \subset Q-G(\widehat{x}) .
$$

Пусть число $\mu>0$ удовлетворяет соотношению

$$
\mu \eta=8\left(1+\left\|G^{\prime}(\widehat{x})\right\|\right) \gamma_{1}\left\|P_{1}\right\|\left(1+\gamma_{2}\left\|\Lambda_{2}\right\|\left\|P_{2}\right\|\right) .
$$

В предыдущем пункте доказательства, при построении отображения $\varphi$, радиусы шаров $\delta_{i}, i=1,2,3,4$, полностью определялись числом $\varepsilon_{1}>0$, которое было подчинено лишь условию $\varepsilon_{1} \gamma_{1}\left\|P_{1}\right\|<1$. Пусть теперь

$$
\varepsilon_{1}=\frac{1}{8 \gamma_{1}\left\|P_{1}\right\|\left(1+\gamma_{2}\left\|\Lambda_{2}\right\|\left\|P_{2}\right\|\right)+2 \mu\|h\|_{X}} .
$$

Ясно, что $\varepsilon_{1} \gamma_{1}\left\|P_{1}\right\|<1$. Далее считаем, что $\delta_{i}, i=1,2,3,4$, определены по данному $\varepsilon_{1}$. Кроме того, считаем, что $\delta_{1}$ столь мало, что если $x \in U_{X}\left(\widehat{x}, \delta_{1}\right)$, то (в силу дифференцируемости $G$ в точке $\widehat{x}$ )

$$
\left\|G(x)-G(\widehat{x})-G^{\prime}(\widehat{x})(x-\widehat{x})\right\|_{Z_{2}} \leqslant \varepsilon_{2}\|x-\widehat{x}\|_{X},
$$

где $\varepsilon_{2}=\min \left(1, \eta /\left(2\|h\|_{X}\right)\right)$.

Положим

$$
\delta_{0}=\min \left(\frac{\delta_{2}}{2 \gamma_{2}\left\|P_{2}\right\|+\mu}, \delta_{3}, \frac{1}{2 \mu}, \frac{\delta_{4}}{\mu\|h\|_{X}}\right) .
$$

Пусть $z \in U_{Z_{1}}\left(0, \delta_{0}\right), z \neq 0$. Согласно свойству микса существует такое $\delta(z)>0$, что для всех $\alpha=\left(\alpha_{1}, \ldots, \alpha_{k}\right) \in \Sigma_{0}^{k}$ 


$$
\begin{aligned}
\| \Phi(\widehat{x}, M(\alpha, \delta(z), \bar{u}))-\sum_{i=1}^{k} \alpha_{i} \Phi\left(\widehat{x}, u_{i}\right) & \\
& =\left\|\Phi(\widehat{x}, M(\alpha, \delta(z), \bar{u}))-\Lambda_{2} \alpha\right\|_{Z_{1}} \leqslant \frac{\|z\|_{Z_{1}}}{2} .
\end{aligned}
$$

Положим $\xi(z)=\widehat{x}+\mu\|z\|_{Z_{1}} h$. Поскольку $\mu\|z\|_{Z_{1}}\|h\|_{X}<\mu \delta_{0}\|h\|_{X} \leqslant \delta_{4}$, то $\xi(z) \in U_{X}\left(\widehat{x}, \delta_{4}\right)$. Положим далее $R(w, z)=R_{2}(w)+\mu\|z\|_{Z_{1}} \beta$, где $\beta=$ $\left(\beta_{1}, \ldots, \beta_{m}, 0, \ldots, 0\right)$ (см. (3.3)).

Пусть $w \in B_{L}\left(0,2\left\|P_{2}\right\|\|z\|_{Z_{1}}\right)$. Тогда $R(w, z) \in U_{\mathbb{R}^{k}}\left(0, \delta_{2}\right) \cap \mathbb{R}_{+}^{k}$, так как

$$
\begin{gathered}
|R(w, z)| \leqslant\left|R_{2}(w)\right|+\mu\|z\|_{Z_{1}}|\beta| \leqslant \gamma_{2}\|w\|_{Z_{1}}+\mu\|z\|_{Z_{1}} \\
\leqslant\left(2 \gamma_{2}\left\|P_{2}\right\|+\mu\right)\|z\|_{Z_{1}}<\left(2 \gamma_{2}\left\|P_{2}\right\|+\mu\right) \delta_{0} \leqslant \delta_{2} .
\end{gathered}
$$

Рассмотрим отображение $\mathscr{F}_{z}: B_{L}\left(0,2\left\|P_{2}\right\|\|z\|_{Z_{1}}\right) \rightarrow L$, заданное формулой

$$
\mathscr{F}_{z}(w)=w+P_{2} z-P_{2} \Phi(\varphi(\xi(z), R(w, z), z, \delta(z)), M(R(w, z), \delta(z), \bar{u})) .
$$

Оно корректно определено (в силу оценок выше) и непрерывно, как суперпозиция непрерывных отображений. Покажем, что это отображение переводит шар $B_{L}\left(0,2\left\|P_{2}\right\|\|z\|_{Z_{1}}\right)$ в себя. Для этого сначала оценим норму разности $\varphi(\xi(z), R(w, z), z, \delta(z))-\xi(z)$ через норму $z$. Из $(3.3)$ и вида функции $R(w, z)$ и оператора $\Lambda_{2}$ следует, что

$$
\Lambda_{1}\left(\mu\|z\|_{Z_{1}} h\right)+\Lambda_{2} R(w, z)-\Lambda_{2} R_{2}(w)=0 .
$$

Используя (3.6), (3.18), (3.8), (3.17), выражение для $\varepsilon_{1}$, оценку для $R_{2}$ и то, что $K_{1} \leqslant 2 \gamma_{1}\left(K_{1}=\gamma_{1} /(1-\theta)=\gamma_{1} /\left(1-\varepsilon_{1} \gamma_{1}\left\|P_{1}\right\|\right)\right.$ и $\left.\varepsilon_{1} \gamma_{1}\left\|P_{1}\right\|<1 / 2\right)$, будем иметь

$$
\begin{aligned}
&\|\varphi(\xi(z), R(w, z), z, \delta(z))-\xi(z)\|_{X} \\
& \leqslant K_{1}\left\|P_{1}\right\|\|\Phi(\xi(z), M(R(w, z), \delta(z), \bar{u}))-z\|_{Z_{1}} \\
&= K_{1}\left\|P_{1}\right\| \| \Phi(\xi(z), M(R(w, z), \delta(z), \bar{u}))-\Phi(\widehat{x}, M(R(w, z), \delta(z), \bar{u})) \\
&-\Lambda_{1}\left(\mu\|z\|_{Z_{1}} h\right)+\Phi(\widehat{x}, M(R(w, z), \delta(z), \bar{u}))-\Lambda_{2} R(w, z)+\Lambda_{2} R_{2}(w)-z \|_{Z_{1}} \\
& \leqslant K_{1}\left\|P_{1}\right\|\left(\varepsilon_{1} \mu\|z\|_{Z_{1}}\|h\|_{X}+\frac{1}{2}\|z\|_{Z_{1}}+\left\|\Lambda_{2} R_{2}(w)\right\|_{Z_{1}}+\|z\|_{Z_{1}}\right) \\
& \leqslant 2 \gamma_{1}\left\|P_{1}\right\|\left(\frac{1}{2}+\frac{1}{2}+2 \gamma_{2}\left\|\Lambda_{2}\right\|\left\|P_{2}\right\|+1\right)\|z\|_{Z_{1}} \\
&= 4 \gamma_{1}\left\|P_{1}\right\|\left(1+\gamma_{2}\left\|\Lambda_{2}\right\|\left\|P_{2}\right\|\right)\|z\|_{Z_{1}} .
\end{aligned}
$$

Отсюда и из вида функции $\xi(\cdot)$ следует, что

$$
\begin{aligned}
& \|\varphi(\xi(z), R(w, z), z, \delta(z))-\widehat{x}\|_{X} \\
& \quad \leqslant\|\varphi(\xi(z), R(w, z), z, \delta(z))-\xi(z)\|_{X}+\mu\|z\|_{Z_{1}}\|h\|_{X} \\
& \quad \leqslant\left(4 \gamma_{1}\left\|P_{1}\right\|\left(1+\gamma_{2}\left\|\Lambda_{2}\right\|\left\|P_{2}\right\|\right)+\mu\|h\|_{X}\right)\|z\|_{Z_{1}} .
\end{aligned}
$$

Применяя $P_{2}$ к обеим частям $(3.13)$, получаем, что $P_{2} \Lambda_{2} R(w, z)=P_{2} \Lambda_{2} R_{2}(w)=$ $w$. Используя это обстоятельство, соотношения (3.8), (3.17), (3.20) и выражение 
для $\varepsilon_{1}$, приходим к неравенству

$$
\begin{aligned}
\left\|\mathscr{F}_{z}(w)\right\|_{Z_{1}} & =\| P_{2} \Phi(\varphi(\xi(z), R(w, z), z, \delta(z)), M(R(w, z), \delta(z), \bar{u})) \\
& -P_{2} \Phi(\widehat{x}, M(R(w, z), \delta(z), \bar{u}))-P_{2} \Lambda_{1}(\varphi(\xi(z), R(w, z), z, \delta(z))-\widehat{x}) \\
& +P_{2} \Phi(\widehat{x}, M(R(w, z), \delta(z), \bar{u}))-P_{2} \Lambda_{2} R(w, z)-P_{2} z \|_{Z_{1}} \\
\leqslant & \left\|P_{2}\right\| \varepsilon_{1}\|\varphi(\xi(z), R(w, z), z, \delta(z))-\widehat{x}\|_{X}+\frac{1}{2}\left\|P_{2}\right\|\|z\|_{Z_{1}}+\left\|P_{2}\right\|\|z\|_{Z_{1}} \\
\leqslant & \frac{3}{2}\left\|P_{2}\right\|\|z\|_{Z_{1}}+\left\|P_{2}\right\| \varepsilon_{1}\left(4 \gamma_{1}\left\|P_{1}\right\|\left(1+\gamma_{2}\left\|\Lambda_{2}\right\|\left\|P_{2}\right\|\right)+\mu\|h\|_{X}\right)\|z\|_{Z_{1}} \\
\leqslant & 2\left\|P_{2}\right\|\|z\|_{Z_{1}},
\end{aligned}
$$

которое означает, что $\mathscr{F}_{z}$ для каждого $z \in B_{Z_{1}}\left(0, \delta_{0}\right), z \neq 0$, непрерывно переводит шар $B_{L}\left(0,2\left\|P_{2}\right\|\|z\|_{Z_{1}}\right)$ в себя. По теореме Брауэра существует точка $\bar{w}(z) \in$ $B_{L}\left(0,2\left\|P_{2}\right\|\|z\|_{Z_{1}}\right)$ такая, что $\mathscr{F}_{z}(\bar{w}(z))=\bar{w}(z)$, или $P_{2} \Phi(\varphi(\xi(z), R(\bar{w}(z), z), z, \delta(z))$, $M(R(\bar{w}(z), z), \delta(z), \bar{u}))=P_{2} z$. Полагая $\alpha(z)=R(\bar{w}(z), z)$, получаем равенство (3.7) при $z \neq 0$. Если обозначить $x(z)=\varphi(\xi(z), \alpha(z), z, \delta(z))$ и $u(z)=$ $M(\alpha(z), \delta(z), \bar{u})$ при $z \neq 0$ и $x(0)=\widehat{x}, u(0)=\widehat{u}$, то из (3.5) и (3.7) следует, как уже было сказано в конце п. 1 , равенство $\Phi(x(z), u(z))=z$, справедливое для всех $z \in W_{0}=U_{Z}\left(0, \delta_{0}\right)$, и при этом $x(z) \in V_{0}=U_{X}\left(\widehat{x}, \delta_{1}\right)$.

Нужная оценка следует из (3.20) с $K=4 \gamma_{1}\left\|P_{1}\right\|\left(1+\gamma_{2}\left\|\Lambda_{2}\right\|\left\|P_{2}\right\|\right)+\mu\|h\|_{X}$.

4. Доказательство включения $G(x(z)) \in Q, z \in U_{Z}\left(0, \delta_{0}\right)$. Считаем, что $z \neq 0$, так как при $z=0$ включение выполнено по условию теоремы. Используя определения $\xi(z), x(z),(3.16)$ (учитывая, что $\left.x(z) \in U_{X}\left(\widehat{x}, \delta_{1}\right)\right)$, (3.19), (3.20), $(3.15)$ и определение $\varepsilon_{2}$, будем иметь

$$
\begin{aligned}
& \left\|G(x(z))-G(\widehat{x})-G^{\prime}(\widehat{x})\left(\mu\|z\|_{Z_{1}} h\right)\right\|_{Z_{2}} \\
& \quad=\left\|G(x(z))-G(\widehat{x})-G^{\prime}(\widehat{x})(x(z)-\widehat{x})+G^{\prime}(\widehat{x})(x(z)-\xi(z))\right\|_{Z_{2}} \\
& \quad \leqslant \varepsilon_{2}\|x(z)-\widehat{x}\|_{X}+\left\|G^{\prime}(\widehat{x})\right\|\|x(z)-\xi(z)\|_{X} \\
& \quad \leqslant \varepsilon_{2} \mu\|z\|_{Z_{1}}\|h\|_{X}+\left(\varepsilon_{2}+\left\|G^{\prime}(\widehat{x})\right\|\right) \cdot 4 \gamma_{1}\left\|P_{1}\right\|\left(1+\gamma_{2}\left\|\Lambda_{2}\right\|\left\|P_{2}\right\|\right)\|z\|_{Z_{1}} \\
& \quad \leqslant \frac{\mu \eta}{2}\|z\|_{Z_{1}}+\frac{\mu \eta}{2}\|z\|_{Z_{1}}=\mu\|z\|_{Z_{1}} \eta .
\end{aligned}
$$

Обозначим $t=t(z)=\mu\|z\|_{Z_{1}}$. Так как $\|z\|_{Z_{1}}<\delta_{0} \leqslant 1 /(2 \mu)$, то $0<t \leqslant 1 / 2$. Положим $\widetilde{z}=t^{-1}\left(G(x(z))-G(\widehat{x})-t G^{\prime}(\widehat{x}) h\right)$. Из (3.21) следует, что $\widetilde{z} \in B_{Z_{2}}(0, \eta)$, но тогда вследствие (3.14) и выпуклости $Q$ получаем, что $G(x(z))-G(\widehat{x})=$ $t\left(G^{\prime}(\widehat{x}) h+\widetilde{z}\right)=(1-t) 0+t\left(G^{\prime}(\widehat{x}) h+\widetilde{z}\right) \in Q-G(\widehat{x})$, т. е. $G(x(z)) \in Q$. Теорема о разрешимости доказана.

\section{4. Дополнение: дальнейшее развитие формализма Лагранжа (Е. Р. Аваков)}

4.1. Введение. Выше было показано, что принцип Лагранжа является универсальным инструментом исследования экстремальных задач, однако его возможности все же не безграничны. Мы продемонстрируем это на примере гладкой задачи с ограничениями типа равенств

$$
f_{0}(x) \rightarrow \min , \quad F(x)=0,
$$


являющейся частным случаем задачи $\left(\mathrm{P}_{1}\right)$. Здесь $X$ и $Y$ - нормированные пространства, $V$ - открытое подмножество $X, f_{0}: V \rightarrow \mathbb{R}$ и $F: V \rightarrow Y$.

Функция Лагранжа задачи $\left(\mathrm{P}_{a}\right)$ имеет вид

$$
\mathscr{L}(x, \bar{\lambda})=\lambda_{0} f_{0}(x)+\langle\lambda, F(x)\rangle,
$$

где $\bar{\lambda}=\left(\lambda_{0}, \lambda\right) \in \mathbb{R} \times Y^{*}$.

Принцип Лагранжа для задачи $\left(\mathrm{P}_{a}\right)$ содержится в следствии 1 и заключается в том, что если $\widehat{x} \in V$ - локальный минимум в данной задаче, то при определенных условиях гладкости и замкнутости образа $\operatorname{Im} F^{\prime}(\widehat{x})$ найдутся, не равные одновременно нулю, множители Лагранжа $\lambda_{0} \geqslant 0$ и $\lambda \in Y^{*}$ такие, что

$$
\mathscr{L}_{x}(\widehat{x}, \bar{\lambda})=0 \Leftrightarrow \lambda_{0} f_{0}^{\prime}(\widehat{x})+\left(F^{\prime}(\widehat{x})\right)^{*} \lambda=0 .
$$

Отсюда видно, что содержателен лишь регулярный случай, когда $\operatorname{Im} F^{\prime}(\widehat{x})=Y$, так как в этой ситуации $\lambda_{0} \neq 0$ и уравнение (4.1) позволяет "квалифицированно" выделить "подозрительные" на экстремум допустимые в задаче $\left(\mathrm{P}_{a}\right)$ точки. В нерегулярном случае, когда $\operatorname{Im} F^{\prime}(\widehat{x}) \neq Y$, равенство (4.1) выполняется автоматически с $\lambda_{0}=0$, независимо от минимизируемого функционала. То есть в этом случае классические необходимые условия первого порядка вырождаются и, тем самым, никакой дополнительной информации о точке $\widehat{x}$, кроме тавтологической, не несут: $\widehat{x}$ - нерегулярная точка ограничений задачи. Но равенство (4.1) есть необходимое условие минимума и поэтому любая нерегулярная точка ограничений является "подозрительной" на экстремум (с точки зрения принципа Лагранжа). При этом мы не можем применить для дальнейшего исследования нерегулярных точек классические условия второго порядка, так как в нерегулярном случае они, вообе говоря, неверны.

Экстремальные задачи, в которых не выполнены условия регулярности, называют, следуя Блиссу (см. [6]), анормальными задачами. В задаче $\left(\mathrm{P}_{a}\right)$ ограничения могут быть и более общего вида (например, можно добавить ограничения типа неравенств и включений). Но для любой такой задачи всегда можно выделить главный, регулярный случай, когда правило множителей Лагранжа дает содержательную информацию о точке экстремума, и нерегулярный, когда (формально выписанные) необходимые условия содержательной информации не дают. Понятно, что свойство анормальности проявляется и в приложениях общей постановки, скажем, к задачам математического программирования, вариационного исчисления и оптимального управления. Для этих задач классические методы исследования, основанные на соответствующих необходимых условиях (правило множителей Лагранжа, уравнение Эйлера-Лагранжа, принцип максимума Понтрягина), не работают, так как не дают никакой информации о точках экстремума.

В работах [16] и [17] впервые были получены результаты, являющиеся естественным развитием формализма Лагранжа на нерегулярный случай. В последующих работах [18] и [19] общие утверждения из [16], [17] были применены к задачам вариационного исчисления и оптимального управления. Затем результаты из [16] и [17] были распространены на более общие классы задач (см. [20]). Здесь мы приведем некоторые результаты из [17], имеющие непосредственное отношение к развитию принципа Лагранжа для задачи $\left(\mathrm{P}_{a}\right)$. Мы 
начинаем с теоремы, которая играет ту же роль при получения содержательных необходимых условий для анормальных задач, что и теорема Люстерника для задачи $\left(\mathrm{P}_{a}\right)$ в регулярном случае (см. [12]).

4.2. Теорема о поправке. Пусть $X$ и $Y$ - нормированные пространства, $U$ - окрестность точки $\widehat{x} \in X$, отображение $F: U \rightarrow Y$ дважды дифференцируемо в $\widehat{x}$ и подпространство $\operatorname{Im} F^{\prime}(\widehat{x})$ замкнуто. Положим $Z=\operatorname{Im} F^{\prime}(\widehat{x}) \times$ $\left(Y / \operatorname{Im} F^{\prime}(\widehat{x})\right)$, и пусть $\pi: Y \rightarrow Y / \operatorname{Im} F^{\prime}(\widehat{x})$ - каноническая проекция.

Для каждого $h \in X$ рассмотрим отображение $G(\widehat{x}, h): X \rightarrow Z$, определенное по формуле

$$
G(\widehat{x}, h) x=\left(F^{\prime}(\widehat{x}) x, \pi F^{\prime \prime}(\widehat{x})[h, x]\right) .
$$

ОПРЕДЕЛЕНИЕ. Если $h \in X$ таково, что

$$
\operatorname{Im} G(\widehat{x}, h)=Z \text {, }
$$

то будем говорить, что отображение $F$ регулярно в точке $\widehat{x}$ по направлению $h$.

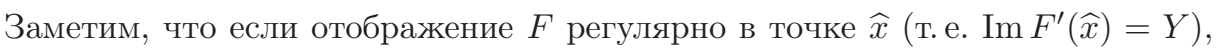
то $F$ регулярно по любому направлению. Обратное, как показывают простые примеры, вообще говоря, неверно.

Напомним, что $U_{X}(\widehat{x}, \rho)$ обозначает открытый шар в нормированном пространстве $X$ с центром в точке $\widehat{x}$ и радиусом $\rho$. Если $A$ - непустое подмножество $X$, то cone $A$ обозначает выпуклую коническую оболочку множества $A$.

Теорема (о поправке). Пусть $X$ и $Y$ - банаховы пространства, $U$ - окрестность точки $\widehat{x} \in X, h \in X$, отображение $F: U \rightarrow Y$ дваждъ дифференцируемо в $\widehat{x}$ и регулярно в точке $\widehat{x}$ по направлению $h$. Тогда найдутся окрестность $V \subset U$ точки $\widehat{x}$, числа $\alpha>0 u K>0$ и отображение $r: V \rightarrow X$ такие, что для любых $x \in V \cap\left(\widehat{x}+\operatorname{cone} B_{X}(h, \alpha)\right), x \neq \widehat{x}, u y \in \operatorname{Im} F^{\prime}(\widehat{x})$ выполняются равенство

$$
F(x+r(x))=F(\widehat{x})
$$

и оченка

$$
\|r(x)\|_{X} \leqslant K\left(\|y\|_{Y}+\frac{\|F(x)-F(\widehat{x})-y\|_{Y}}{\|x-\widehat{x}\|_{X}}\right) .
$$

Это теорема является усилением упомянутой теоремы Люстерника, которая формулируется для регулярного случая $\left(\operatorname{Im} F^{\prime}(\widehat{x})=Y\right)$. Действительно, если $y=F(x)-F(\widehat{x})$ и $h=0$, то соотношения (4.2) и (4.3) переходят в известные утверждения теоремы Люстерника (см. [12]).

В то же время данная теорема, в нерегулярном случае, дает содержательную оценку расстояния до поверхности уровня отображения $F$ в точке $\widehat{x}$. Это уже видно из простого примера: $F\left(x_{1}, x_{2}\right)=x_{1}^{2}-x_{2}^{2}, \widehat{x}=(0,0)$.

Сформулированная теорема имеет ясное геометрическое следствие, дающее описание касательного конуса ко множеству $M=\{x \in U \mid F(x)=F(\widehat{x})\}$ в точке $\widehat{x}$. Напомним, что элемент $h \in X$ называется касательным вектором $\kappa M$ в точке $\widehat{x} \in M$, если существуют $\varepsilon>0$ и отображение $r:(-\varepsilon, \varepsilon) \rightarrow X$ такие, что $\widehat{x}+t h+r(t) \in M$ для всех $t \in(-\varepsilon, \varepsilon)$, где $r(t)=o(t)$ при $t \rightarrow 0$. Множество всех касательных векторов к $M$ в точке $\widehat{x} \in M$ образует конус, который называется касательным конусом к $M$ в точке $\widehat{x}$ и обозначается $T_{\widehat{x}} M$. 
Положим

$$
\begin{aligned}
H(\widehat{x}) & =\left\{h \in \operatorname{Ker} F^{\prime}(\widehat{x}) \mid F^{\prime \prime}(\widehat{x})[h, h] \in \operatorname{Im} F^{\prime}(\widehat{x})\right\} \\
H_{0}(\widehat{x}) & =\{h \in H(\widehat{x}) \mid \operatorname{Im} G(\widehat{x}, h)=Z\} .
\end{aligned}
$$

СлеДСтвиЕ. В условиях теоремы

$$
H_{0}(\widehat{x}) \subset T_{\widehat{x}} M \subset H(\widehat{x})
$$

ДокАзАТЕльство. Правое включение есть простое следствие определения касательного вектора и дважды дифференцируемости отображения $F$ в точке $\widehat{x}$.

Докажем левое включение. Пусть $h \in H_{0}(\widehat{x})$. Для каждого $t>0$ положим $x(t)=\widehat{x}+t h$ и $y(t)=t^{2} F^{\prime \prime}(\widehat{x})[h, h]$. Тогда из теоремы о поправке следует, что для достаточно малого $\varepsilon>0$ существует отображение $r_{1}:(0, \varepsilon) \rightarrow X$ такое, что $F\left(\widehat{x}+t h+r_{1}(t)\right)=F(\widehat{x})$ и $r_{1}(t)=o(t)$ при $t \rightarrow 0$. Так как, очевидно, $-h$ также принадлежит $H_{0}(\widehat{x})$, то аналогично получаем существование отображения $r_{2}:(-\varepsilon, 0) \rightarrow X$ такого, что $F\left(\widehat{x}+t h+r_{2}(t)\right)=F(\widehat{x})$ и $r_{2}(t)=o(t)$ при $t \rightarrow 0$. Отсюда сразу следует, что $h \in T_{\widehat{x}} M$. Следствие доказано.

Заметим, что в регулярном случае, когда $\operatorname{Im} F^{\prime}(\widehat{x})=Y$, получаем, что $H_{0}(\widehat{x})=H(\widehat{x})=\operatorname{Ker} F^{\prime}(\widehat{x})$, т. е. утверждение следствия превращается в "геометрическую часть" теоремы Люстерника: $T_{\widehat{x}} M=\operatorname{Ker} F^{\prime}(\widehat{x})$.

В нерегулярном случае данное следствие дает содержательное описание касательного конуса. Это видно уже из простейших примеров. Например, пусть $F\left(x_{1}, x_{2}\right)=x_{1}^{2}-x_{2}^{2}+g\left(x_{1}, x_{2}\right)$, где $g(0,0)=0$ и $g^{\prime}(0,0)=0$. Тогда, если $M=\left\{\left(x_{1}, x_{2}\right) \in \mathbb{R}^{2} \mid F\left(x_{1}, x_{2}\right)=F(0,0)\right\}$, то из следствия вытекает, что $T_{(0,0)} M=\left\{\left(x_{1}, x_{2}\right) \in \mathbb{R}^{2} \mid x_{1}^{2}-x_{2}^{2}=0\right\}$. Можно привести и более сложные примеры конечномерных и бесконечномерных отображений $F$, иллюстрирующих возможности теоремы о поправке и ее следствия, но это выходит за рамки данной работы.

Доказательство теоремы о поправке содержится в [17], и здесь мы его не приводим.

4.3. Необходимые условия первого порядка для задачи $\left(\mathrm{P}_{a}\right)$. Для исследования анормальных экстремальных задач вида $\left(\mathrm{P}_{a}\right)$ в работе [16] была введена следующая обобщенная функция Лагранжа

$$
\mathscr{L}^{a}(x, \bar{\lambda}, h)=\lambda_{0} f_{0}(x)+\left\langle\lambda_{1}, F(x)\right\rangle+\left\langle\lambda_{2}, F^{\prime}(x) h\right\rangle
$$

где $\bar{\lambda}=\left(\lambda_{0}, \lambda_{1}, \lambda_{2}\right) \in \mathbb{R} \times Y^{*} \times Y^{*}$.

ТЕорема (необходимые условия минимума первого порядка для задачи $\left(\mathrm{P}_{a}\right)$ ). Пусть $\widehat{x} \in V$ - локальный минимум в задаче $\left(\mathrm{P}_{a}\right)$. Тогда, если в этой задаче $X$ и $Y$ - банаховы пространства, функиия $f_{0}$ дифферениируема в $\widehat{x}$, отображение $F$ дважды дифферениируемо в $\widehat{x}$, то для каждого $h \in H(\widehat{x})$, для которого подпространство $\operatorname{Im} G(\widehat{x}, h)$ замкнуто, найдутся множители Лагранжа 


$$
\begin{gathered}
\lambda_{0}=\lambda_{0}(h) \geqslant 0, \lambda_{1}=\lambda_{1}(h) \text { u } \lambda_{2}=\lambda_{2}(h) \text { maкue, umo } \\
\lambda_{0}+\left\|\lambda_{2}\right\| \neq 0, \\
L_{x}^{a}(\widehat{x}, \bar{\lambda}, h)=0 \Leftrightarrow \begin{array}{c}
\lambda_{0} f_{0}^{\prime}(\widehat{x})+\left(F^{\prime}(\widehat{x})\right)^{*} \lambda_{1}+\left(F^{\prime \prime}(\widehat{x}) h\right)^{*} \lambda_{2}=0, \\
\left(F^{\prime}(\widehat{x})\right)^{*} \lambda_{2}=0 .
\end{array}
\end{gathered}
$$

ДоказАтельство. 1. Регулярный по направлению случай. Пусть $h \in H(\widehat{x})$ и $\operatorname{Im} G(\widehat{x}, h)=Z$, т. е. $h \in H_{0}(\widehat{x})$. Легко видеть, что $G(\widehat{x}, h)$ есть производная в точке $h$ отображения $\Phi: X \rightarrow Z$, определенного формулой

$$
\Phi(x)=\left(F^{\prime}(\widehat{x}) x, \pi\left(\frac{1}{2} F^{\prime \prime}(\widehat{x})[x, x]\right)\right)
$$

и значит, $h$ - регулярная точка этого отображения. Согласно теореме Люстерника, если $h_{0} \in \operatorname{Ker} G(\widehat{x}, h)$, то найдутся $\varepsilon>0$ и отображение $r:(-\varepsilon, \varepsilon) \rightarrow x$, $r(t)=o(t)$ при $t \rightarrow 0$, такие, что

$$
\Phi\left(h+t h_{0}+r(t)\right)=\Phi(h)=0
$$

для всех $t \in(-\varepsilon, \varepsilon)$.

Положим $h(t)=h+t h_{0}+r(t)$. Тогда из соотношений (4.7) и (4.8) следует, что $h(t) \in H(\widehat{x})$. Кроме того (уменьшая, если необходимо, $\varepsilon$ ), можно считать, что $\operatorname{Im} G(\widehat{x}, h(t))=Z$ (это следствие хорошо известного и нетрудно проверяемого факта, что множество всех сюръективных линейных непрерывных операторов из одного банахова пространства в другое открыто в соответствующей операторной топологии) и, значит, $h(t) \in H_{0}(\widehat{x})$. Тогда согласно следствию $h(t) \in T_{\widehat{x}} M$.

Поскольку $\widehat{x}$ - решение задачи $\left(\mathrm{P}_{a}\right)$, то на любом векторе из $T_{\widehat{x}} M$ функционал $f_{0}^{\prime}(\widehat{x})$ равен нулю и поэтому

$$
0=\left\langle f_{0}^{\prime}(\widehat{x}), h(t)\right\rangle=\left\langle f_{0}^{\prime}(\widehat{x}), h+t h_{0}+r(t)\right\rangle=\left\langle f_{0}^{\prime}(\widehat{x}), h_{0}\right\rangle+\left\langle f_{0}^{\prime}(\widehat{x}), r(t)\right\rangle .
$$

Отсюда, деля на $t$ и переходя к пределу при $t \rightarrow 0$, получаем, что $\left\langle f_{0}^{\prime}(\widehat{x}), h_{0}\right\rangle=0$. Поскольку это верно для любого вектора $h_{0} \in \operatorname{Ker} G(\widehat{x}, h)$, то по лемме об аннуляторе ядра регулярного оператора $f_{0}^{\prime}(\widehat{x}) \in \operatorname{Im}(G(\widehat{x}, h))^{*}$. Следовательно, найдутся элементы $y_{1}^{*} \in\left(\operatorname{Im} F^{\prime}(\widehat{x})\right)^{*}$ и $v^{*} \in\left(Y / \operatorname{Im} F^{\prime}(\widehat{x})\right)^{*}$ такие, что

$$
f_{0}^{\prime}(\widehat{x})+\left(F^{\prime}(\widehat{x})\right)^{*} y_{1}^{*}+\left(\pi F^{\prime \prime}(\widehat{x}) h\right)^{*} v^{*}=0 .
$$

Пусть $\lambda_{1}$ - какое-нибудь продолжение (согласно теореме Хана-Банаха) функционала $y_{1}^{*}$ на все $Y$, а $\lambda_{2}=\pi^{*} v^{*}$. Тогда получаем утверждения (4.4) и (4.5) теоремы с $\lambda_{0}=1$. Утверждение (4.6) легко следует из определения $v^{*}$.

2. Нерегулярный по направлению случай. Пусть $h \in H(\widehat{x})$ и $\operatorname{Im} G(\widehat{x}, h) \neq Z$. Так как $\operatorname{Im} G(\widehat{x}, h)$ - замкнутое подпространство, то у него существует нетривиальный аннулятор, т. е. такие функционалы $y_{1}^{*} \in\left(\operatorname{Im} F^{\prime}(\widehat{x})\right)^{*}$ и $v^{*} \in\left(Y / \operatorname{Im} F^{\prime}(\widehat{x})\right)^{*}$, не равные одновременно нулю, что

$$
\left(F^{\prime}(\widehat{x})\right)^{*} y_{1}^{*}+\left(\pi F^{\prime \prime}(\widehat{x}) h\right)^{*} v^{*}=0 .
$$

Повторяя предыдущие рассуждения, получаем утверждения (4.5) и (4.6) теоремы с $\lambda_{0}=0$. Осталось проверить, что $\lambda_{2} \neq 0$. Действительно, если это так, то $v^{*}=0$, а тогда из (4.10) следует, что $y_{1}^{*}=0$, что невозможно. Теорема доказана. 


\section{5. Исторический комментарий}

Первый намек, как уже было сказано во введении, на вид необходимых условий экстремума в задаче без ограничений содержится в письме Ферма, адресованном Декарту (1638 г.) [2], хотя понятие производной здесь еще не фигурирует. Основы дифференциального исчисления впервые были изложены лишь в 1684 г. в работе Лейбница [21]. Конечномерный вариант следствия 1 для задачи с равенствами сформулирован Лагранжем (1797 г.) [5]. Регулярный случай следствия 1 для задачи с равенствами (когда $\mathscr{F}^{\prime}(\widehat{x}) X=Y$ ) был доказан Л. А. Люстерником (1934 г.) [22] и передоказан с некоторым усилением Л. Грейвсом [23]. Следствия 1 и 2 в конечномерном случае были получены в диссертации В. Каруша [8], защищенной в Чикаго в 1939 г., но остававшейся неизвестной за пределами узкого круга специалистов. Конечномерный вариант следствия 1 был передоказан Ф. Джоном в 1948 г. [24], а следствия 2 - Куном и Таккером [25]. Вариационное исчисление, как также было сказано, ведет свою историю с задачи о брахистохроне, поставленной И. Бернулли в 1696 г. [3]. Начала этого исчисления были разработаны Эйлером. Затем к этим исследованиям подключился Лагранж, разработавший метод вариаций для вариационных задач с ограничениями, называемый сегодня правилом множителей Лагранжа. Доказательство этого правила стало возможными лишь после того, как были сформулированы и доказаны теоремы о неявной и обратной функциях (первая публикация по этому поводу принадлежит У. Дини [26]). На современном уровне строгости доказательство правила множителей Лагранжа для изопериметрической задачи дано К. Вейерштрассом [27]; считается, что первое строгое доказательство правила множителей Лагранжа для задачи Лагранжа принадлежит Майеру [28]. В XX в. стали появляться учебники по вариационному исчислению, и важную роль здесь сыграла книга Больца [29]. Первым учебником по вариационному исчислению в Московском университете была книга Д. Ф. Егорова [30]. Итоги развития теории вариационного исчисления к концу сороковых годов были подведены в монографии Блисса [6]. В шестидесятые годы вышла книга И. М. Гельфанда и С. В. Фомина [31], основанная на лекциях Гельфанда, читавшихся им в 1956 г. Все описанные результаты о необходимых условиях экстремума ("первого порядка", как иногда говорят) в задачах вариационного исчисления вытекают из следствия 1. Разработка теории оптимального управления началась с семинара Л. С. Понтрягина. Итоги ее развития к концу пятидесятых годов подведены в монографии [10] (хотя первая задача, которую следует отнести к оптимальному управлению, была сформулирована и решена Ньютоном [1]). Принцип максимума для задач оптимального управления, линейных по фазовым переменным, был доказан Р. В. Гамкрелидзе [10]. Первое доказательство принципа максимума Понтрягина в нетривиальном случае принадлежит В. Г. Болтянскому [10]. Теория ляпуновских задач опирается на теорему о векторных мерах А. А. Ляпунова [9]. Общая теория необходимых условий экстремума началась с работы А.Я. Дубовицкого и А.А. Милютина [11]. Ее началам посвящены книги [32], [12], [33]. В дополнении к настоящей 
статье (раздел 4) представлено дальнейшее развитие теории экстремума в духе лагранжева формализма для нерегулярных (анормальных, по определению Блисса, см. [6]) задач.

\section{Список литературы}

[1] И. Ньютон, Математические начала натуральной философии, перевод, прим. и поясн. А.Н. Крылова, Собрание трудов академика А.Н. Крылова, VII, Изд-во AH CCCP, M.-Л., 1936, 659 с.; пер. с лат.: I. Newton, Philosophice naturalis principia mathematica, William Dawson \& Sons, Ltd., London, 1687, viii $+510+\mathrm{i}$ pp.

[2] П. Ферма, "Метод отыскания наибольших и наименьших значений", в кн.: Р. Декарт, Геометрия, с приложением избранных работ П. Ферма и переписки Декарта, ОНТИ, М.-Л., 1938, 154-155; пер. с фр.: Р. Fermat, "Méthode pour la recherche du minimum et du maximum", Euvres de Fermat, v. 1, Gauthier-Villars, Paris, 1891.

[3] И. Бернулли, "Новая задача, к разрешению которой приглашаются математики", в кн.: И. Бернулли, Избранные сочинения по механике, ОНТИ, М.-Л., 1937, 19-20; пер. с лат.: Joh. Bernoulli, "Problema novum ad cujus solutionem Mathematici invitantur", Acta eruditorum, 15 (1696), 264-269.

[4] Л. Эйлер, Метод нахождения кривых линий, обладающих свойствами максимума, либо минимума, или решение изопериметрической задачи, взятой в самом широком смысле, ГТТИ, М.-Л., 1934, 600 с.; пер. с лат.: L. Euler, Methodus inveniendi lineas curvas maximi minimive proprietate gaudentes sive solutio problematis isoperimetrici latissimo sensu accepti, Bousquet, Lausanne, 1744, 322 pp.

[5] J.L. Lagrange, Théorie des fonctions analytiques, L'Imprimerie de la République, Paris, 1797, 277 pp.

[6] Г.А. Блисс, Лекции по вариационному исчислению, ИЛ, Л., 1950, 347 с.; G. A. Bliss, Lectures on the calculus of variations, Univ. of Chicago Press, Chicago, IL, 1946, ix+296 pp.

[7] Л.В. Канторович, Математические методы организачии и планирования производства, Ленингр. ун-т, Л., 1939, 68 с.; англ. пер.: L. V. Kantorovich, "Mathematical methods of organizing and planning production", Management Sci., 6 (1959/1960), 366-422.

[8] W.E. Karush, Minima of functions of several variables with inequalities as side conditions, Univ. of Chicago, 1939, 25 pp.

[9] А. А. Ляпунов, "О вполне аддитивных вектор-функциях", Изв. АН СССР. Сер. матем., 4:6 (1940), 465-478.

[10] Л. С. Понтрягин, В. Г. Болтянский, Р. В. Гамкрелидзе, Е. Ф. Мищенко, Математическая теория оптимальных процессов, Физматлит, М., 1961, 391 с.; англ. пер.: L.S. Pontryagin, V. G. Boltyanskii, R. V. Gamkrelidze, E. F. Mishchenko, The mathematical theory of optimal processes, Interscience Publishers, John Wiley \& Sons, Inc., New York-London, 1962, viii+360 pp.

[11] А.Я. Дубовицкий, А.А. Милютин, "Задачи на экстремум при наличии ограничений", Журн. вычисл. матем. и матем. физ., 5:3 (1965), 395-453; англ. пер.: A. Ya. Dubovitskii, A. A. Milyutin, "Extremum problems in the presence of restrictions", U.S.S.R. Comput. Math. Math. Phys., 5:3 (1965), 1-80.

[12] А.Д. Иоффе, В. М. Тихомиров, Теория экстремальных задач, Наука, М., 1974, 479 с.; англ. пер.: A.D. Ioffe, V. M. Tihomirov, Theory of extremal problems, Stud. Math. Appl., 6, North-Holland Publishing Co., Amsterdam-New York, 1979, xii+460 pp.

[13] Г. Г. Магарил-Ильяев, В. М. Тихомиров, "Метод Ньютона, дифференциальные уравнения и принцип Лагранжа для необходимых условий экстремума", Onmuмальное управление, Сборник статей. K 60-летию со дня рождения профессора 
Виктора Ивановича Благодатских, Тр. МИАН, 262, МАИК, М., 2008, 156-177; англ. пер.: G. G. Magaril-Il'yaev, V. M. Tikhomirov, "Newton's method, differential equations, and the Lagrangian principle for necessary extremum conditions", Proc. Steklov Inst. Math., 262 (2008), 149-169.

[14] Ж.-П. Обен, И. Экланд, Прикладной нелинейный анализ, Мир, М., 1988, 512 с.; пер. с англ.: J.-P. Aubin, I. Ekeland, Applied nonlinear analysis, Pure Appl. Math. (N. Y.), A Wiley-Interscience Publication. John Wiley \& Sons, Inc., New York, 1984, $\mathrm{xi}+518 \mathrm{pp}$.

[15] J.F. Bonnans, A. Shapiro, Perturbation analysis of optimization problems, Springer Ser. Oper. Res., Springer-Verlag, New York, 2000, xviii+601 pp.

[16] Е.Р. Аваков, "Условия экстремума для гладких задач с ограничениями типа равенств", Журн. вычисл. матем. и матем. физ., 25:5 (1985), 680-693; англ. пер.: E. R. Avakov, "Extremum conditions for smooth problems with equality-type constraints", U.S.S.R. Comput. Math. Math. Phys., 25:3 (1985), 24-32.

[17] Е. Р. Аваков, "Необходимые условия экстремума для гладких анормальных задач с ограничениями типа равенств и неравенств", Матем. заметки, 45:6 (1989), 3-11; англ. пер.: E. R. Avakov, "Necessary extremum conditions for smooth anormal problems with equality- and inequality-type constraints", Math. Notes, 45:6 (1989), 431-437.

[18] Е. Р. Аваков, "Необходимые условия минимума для нерегулярных задач в банаховых пространствах. Принцип максимума для анормальных задач оптимального управления", Оптимальное управление и дифференииальные игры, Тр. МИАН CCCP, 185, Наука, M., 1988, 3-29; англ. пер.: E. R. Avakov, "Necessary conditions for a minimum for nonregular problems in Banach spaces. The maximum principle for abnormal optimal control problems", Proc. Steklov Inst. Math., 185:2 (1990), 1-32.

[19] Е.Р. Аваков, "Необходимые условия первого порядка для анормальных задач вариационного исчисления", Дифферени. уравнения, 27:5 (1991), 739-745; англ. пер.: E. R. Avakov, "Necessary first-order conditions for anormal variational-calculus problems", Differential Equations, 27:5 (1991), 495-500.

[20] Е.Р. Аваков, А. В. Арутюнов, А.Ф. Измаилов, "Необходимые условия экстремума в задаче математического программирования", Динамические системы и оптимизация, Сборник статей. K 70-летию со дня рождения академика Дмитрия Викторовича Аносова, Тр. МИАН, 256, Наука, М., 2007, 6-30; англ. пер.: E. R. Avakov, A.V. Arutyunov, A.F. Izmailov, "Necessary conditions for an extremum in a mathematical programming problem", Proc. Steklov Inst. Math., 256:1 (2007), 2-25.

[21] G. Leibniz, "Nova methodus pro maximis et minimis itemque tangentibus, quae nec fractas nec irrationales quantitates moratur, et singulare pro illis calculi genus", Acta eruditorum, III (1684), 467-473.

[22] Л.А. Люстерник, “Об условных экстремумах функционалов", Матем. сб., 41:3 (1934), 390-401.

[23] L. M. Graves, "Some mapping theorems", Duke Math. J., 17 (1950), 111-114.

[24] F. John, "Extremum problems with inequalities as subsidiary conditions", Studies and Essays, Presented to R. Courant on his 60th birthday, Jan. 8, 1948, Interscience Publishers, Inc., New York, NY, 1948, 187-204.

[25] H.W. Kuhn, A.W. Tucker, "Nonlinear programming", Proceedings of the Second Berkeley Symposium on Mathematical Statistics and Probability (California, 1950), Univ. of California Press, Berkeley-Los Angeles, 1951, 481-492.

[26] U. Dini, Analisi infinitesimale: Calcolo integrale, Lezzione dettate nella R. Universitá di Pisa nell'anno accademico 1877/78, 2, Autografia Bertini, Pisa, 1878.

[27] K. Weierstrass, Mathematische Werke, v. VII: Vorlesungen über Variationsrechnung, Akademische Verlagsgesellschaft, Leipzig, 1927, vi+324 pp. 
[28] A. Mayer, "Begründung der Lagrange'schen Multiplicatorenmethode in der Variationsrechnung", Math. Ann., 26:1 (1886), 74-82.

[29] O. Bolza, Vorlesungen über Variationsrechnung, G. Teubner, Berlin-Leipzig, 1909, iv $+300+10 \mathrm{pp}$.

[30] Д. Ф. Егоров, Конспект лекций по вариационному исчислению, читанных в Императорском Московском университете в 1912 году, М., 1916.

[31] И. М. Гельфанд, С.В. Фомин, Вариационное исчисление, Физматгиз, М., 1961, 228 с.; перераб. англ. изд.: I. M. Gelfand, S. V. Fomin, Calculus of variations, Prentice-Hall, Inc., Englewood Cliffs, NJ, 1963, vii+232 pp.

[32] И. В. Гирсанов, Лекиии по математической теории экстремалъных задач, Издво Моск. ун-та, М., 1970, 120 с.; англ. пер.: I. V. Girsanov, Lectures on mathematical theory of extremum problems, Lecture Notes in Econom. and Math. Systems, 67, ed. B. T. Poljak, Springer-Verlag, Berlin-New York, 1972, iv+136 pp.

[33] Э. М. Галеев, М. И. Зеликин, С. В. Конягин, Г. Г. Магарил-Ильяев, Н. П. Осмоловский, В. Ю. Протасов, В. М. Тихомиров, А. В. Фурсиков, Оптимальное управление, Изд-во МЦНМО, М., 2008, 320 с.

Е. Р. Аваков (E. R. Avakov)

Институт проблем управления

им. В. А. Трапезникова РАН

E-mail: era@maxmin.ru

\section{Г. Г. Магарил-Ильяев (G. G. Magaril-Il'yaev)}

Московский государственный университет

им. М. В. Ломоносова;

Институт проблем передачи информации

им. А. А. Харкевича РАН

E-mail: magaril@mech.math.msu.su

В. М. Тихомиров (V.M. Tikhomirov)

Московский государственный университет

им. М.В. Ломоносова

E-mail: tikhomir@mccme.ru
Поступила в редакцию

11.10.2012 